\title{
Variational Problems on Vector Bundles
}

\author{
Jürg Fröhlich and Michael Struwe \\ Departments of Mathematics and Physics, Eidgenössische Technische Hochschule, \\ CH-8093 Zürich, Switzerland
}

\begin{abstract}
A variety of problems in quantum physics and classical statistical mechanics, in particular the quantization of topological solitons and the statistical mechanics of defects in ordered media, are described. These problems can be studied within a semi-classical approximation, or with the help of low-temperature expansions, respectively. The calculation of the leading term in such expansions gives rise to variational problems for sections of vector bundles characterized by certain topological constraints. Examples of such problems are the quantization of kinks in the two-dimensional $\lambda \varphi^{4}$ theory and the analysis of Bloch walls in a Landau-Ginzburg model of a threedimensional anisotropic ferromagnet. We state a general existence result for variational problems of this kind and develop regularity and decay estimates for solutions of the Landau-Ginzburg model describing Bloch walls with prescribed boundaries. For certain boundary configurations stability results are established. The relation between the minimizers of the Landau-Ginzburg model in a certain strong-coupling limit and minimal surfaces is pursued in some detail. An open question is whether, asymptotically, the stability of the limit (minimal) surface will imply the stability of the minimizers of the LandauGinzburg model.
\end{abstract}

\section{Contents}

1. Background from Quantum Field Theory and Statistical Mechanics. . . . . . . 431

2. General Existence Results . . . . . . . . . . . . . . . . . . . . . . 444

3. Regularity Results for the Landau-Ginzburg Model. . . . . . . . . . . . . 447

4. Connections with Minimal Surface Theory . . . . . . . . . . . . . . . . 453

5. Uniqueness and Stability of Minimizers . . . . . . . . . . . . . . . . . 458

\section{Background from Quantum Field Theory and Statistical Mechanics}

The variational problems studied in this paper arise in the analysis of configurations of topological defects in ordered media, [1]. As a first example, we consider 
a quantum field theory with topological solitons: The leading contributions in a semiclassical expansion of Euclidean Green functions of soliton fields can be analyzed in terms of classical field configurations with topological defects minimizing an action functional on a space of sections of some vector bundle. A second class of examples concerns ordered media in equilibrium statistical mechanics at low temperatures: The geometry of interfaces in ferromagnets can be understood, to leading order in a low temperature expansion, by minimizing some Landau-Ginzburg energy functional constrained by suitably chosen boundary conditions. A problem dual (in the sense of Kramers-Wannier duality [2]) to the one just described is the analysis of Wilson loop expectations in strongly coupled pure gauge theories in the confinement phase [3]. The chromo-electric flux sheets bounded by Wilson loops take the place of Bloch walls in ferromagnets. The Wilson loops correspond to certain topological constraints imposed on field configurations dual to gauge fields, and the geometry of flux sheets is obtained, to leading order in a strong coupling expansion, by minimizing some action functional in the presence of those topological constraints, i.e. by solving a variational problem on a space of sections of some vector bundle.

It may be clear from these remarks that there is an intimate relation between the problems studied in this paper and problems concerning minimal-length geodesics and minimal surfaces; see Sect. 4. Before we begin with our mathematical analysis of variational problems, we wish to discuss the physical problems briefly described above (which have partly motivated this work) in some more detail. Our presentation is heuristic, although there are plenty of mathematically rigorous results in the literature.

Example 1. (Topological solitons in two space-time-dimensional quantum field models.) We consider a field theory in two space-time dimensions with kink (solitary wave) solutions, such as the $\lambda \varphi_{2}^{4}$-theory. The field equation of the $\lambda \varphi_{2}^{4}$ theory is

$$
\square \varphi(\mathbf{x}, t)-\lambda\left(\varphi(\mathbf{x}, t)^{2}-\xi\right) \varphi(\mathbf{x}, t)=0,(\mathbf{x}, t) \in \mathbb{R} \times \mathbb{R},
$$

where $\square=\partial_{t}^{2}-\partial_{\mathbf{x}}^{2}$ and $\varphi: \mathbb{R}^{2} \rightarrow \mathbb{R}$. (We write $\mathbf{x}$ for the spatial variable even though in this model $\mathbf{x}$ is just a 1-dimensional "vector.") This equation is equivalent to Hamiltonian equations of motion derived from the Hamilton functional

$$
H=\int_{-\infty}^{+\infty} \frac{1}{2}\left[\pi(\mathbf{x})^{2}+(\nabla \varphi)^{2}(\mathbf{x})+\frac{\lambda}{2}\left(\varphi(\mathbf{x})^{2}-\xi\right)^{2}\right] d \mathbf{x},
$$

where $\pi(\mathbf{x})=\left(\partial_{\mathrm{t}} \varphi\right)(\mathbf{x}, t=0)$ is the momentum variable canonically conjugate to $\varphi(\mathbf{x})$ $=\varphi(\mathbf{x}, t=0)$. In classical field theory, the Poisson brackets between $\varphi$ and $\pi$ are given by

$$
\{\varphi(\mathbf{x}), \varphi(\mathbf{y})\}=\{\pi(\mathbf{x}), \pi(\mathbf{y})\}=0,\{\pi(\mathbf{x}), \varphi(\mathbf{y})\}=\delta(\mathbf{x}-\mathbf{y}) .
$$

Assuming that the constants $\lambda$ and $\xi$ in (1.1) and (1.2) are positive, one shows quite easily that the space of finite-energy solutions, i.e., solutions on which $H$ is finite, decomposes into four disjoint components, $\Gamma_{+}, \Gamma_{-}, \Gamma_{s}$, and $\Gamma_{\bar{s}}$, where

$$
\Gamma_{ \pm}=\left\{\varphi(\mathbf{x}, t): \lim _{|\mathbf{x}| \rightarrow \infty} \varphi(\mathbf{x}, t)= \pm \sqrt{\xi}, \text { for all } t\right\}
$$


and

$$
\begin{aligned}
& \Gamma_{s}=\left\{\varphi(\mathbf{x}, t): \lim _{x \rightarrow \infty} \varphi(\mathbf{x}, t)=-\lim _{\mathbf{x} \rightarrow-\infty} \varphi(\mathbf{x}, t)=\sqrt{\xi}, \text { for all } t\right\}, \\
& \Gamma_{\bar{s}}=\left\{\varphi(\mathbf{x}, t): \lim _{\mathbf{x} \rightarrow \infty} \varphi(\mathbf{x}, t)=-\lim _{\mathbf{x} \rightarrow-\infty} \varphi(\mathbf{x}, t)=-\sqrt{\xi}, \text { for all } t\right\} .
\end{aligned}
$$

The minimizing configurations for the Hamilton functional $H$ restricted to $\Gamma_{ \pm}$are

$$
\varphi_{ \pm}(\mathbf{x}) \equiv \pm \sqrt{\xi}, \quad \pi_{ \pm}(\mathbf{x}) \equiv 0
$$

with

$$
H\left(\varphi_{ \pm}, \pi_{ \pm}\right)=0
$$

and the minimizers for $H$ restricted to $\Gamma_{s}, \Gamma_{\bar{s}}$, respectively are

$$
\begin{gathered}
\varphi_{s}(\mathbf{x})=\sqrt{\xi} \tanh \left(\sqrt{\frac{\lambda \xi}{2}} \mathbf{x}-\mathbf{a}\right)=-\varphi_{s}(\mathbf{x}), \\
\pi_{s}(\mathbf{x})=\pi_{\bar{s}}(\mathbf{x}) \equiv 0,
\end{gathered}
$$

where $\mathbf{a}$ is an arbitrary constant. The energy of the minimizers is given by

$$
H\left(\varphi_{s}, \pi_{s}\right)=H\left(\varphi_{\bar{s}}, \pi_{\bar{s}}\right)=\frac{2 \sqrt{2}}{3} \xi \sqrt{\lambda \xi}
$$

The solutions $\varphi_{s}$ and $\varphi_{\bar{s}}$ are static (or standing) kink solutions. Travelling kinks are obtained by applying Lorentz transformations to $\varphi_{s}$ and $\varphi_{\bar{s}^{*}}$. All this is well-known classical field theory.

Next, we wish to consider the quantum theory associated with the field equation (1.1). Quantization consists in replacing Poisson brackets by commutators, multiplied by $(-i)$. Thus one requires

$$
[\varphi(\mathbf{x}), \varphi(\mathbf{y})]=[\pi(\mathbf{x}), \pi(\mathbf{y})]=0,[\pi(\mathbf{x}), \varphi(\mathbf{y})]=-i \delta(\mathbf{x}-\mathbf{y}) .
$$

Note that, formally, $\pi(\mathbf{x})=-i(\partial / \partial \varphi(\mathbf{x}))$; the functional derivative with respect to $\varphi(\mathbf{x})$.

The problem is to find a representation of these commutation relations on some separable Hilbert space $\mathscr{H}$, with the property that a renormalized version of Eq. (1.1) holds in the sense of an equation for operator-valued tempered distributions on $\mathscr{H}$, with $\varphi(\mathbf{x}, t)$ given by $e^{i t H} \varphi(\mathbf{x}) e^{-i t H}$, where $H$ is the Hamilton operator of the quantum theory (Heisenberg picture). This problem has been solved between 1968 and 1976; see [4] for a summary of results and references to the original literature. The upshot of the work that has gone into this problem is that, for $\lambda$ sufficiently small and $\xi=O(1 / \lambda)$, there are four inequivalent representations of (1.8) on Hilbert spaces $\mathscr{H}_{+}, \mathscr{H}_{-}, \mathscr{H}_{s}$, and $\mathscr{H}_{\bar{s}}$ with the following properties: The spaces $\mathscr{H}_{+}$and $\mathscr{H}_{-}$are vacuum sectors of the theory, in the sense that they contain unit vectors $\Omega_{+}, \Omega_{-}$, respectively, which are ground states for the renormalized Hamilton operator

$$
H=\int_{-\infty}^{+\infty} \frac{1}{2}\left[: \pi(\mathbf{x})^{2}:+:(\nabla \varphi)^{2}(\mathbf{x}):+\frac{\lambda}{2}:\left(\varphi(\mathbf{x})^{2}-\xi\right)^{2}:+\varepsilon\right] d \mathbf{x}
$$


(In (1.9) the double colons indicate standard Wick ordering [4], and $\varepsilon$ is a constant chosen such that $H$ is a positive operator with $\inf \operatorname{spec} H_{\mid \mathscr{H}_{ \pm}}=0$.) The ground state vectors $\Omega_{+}$and $\Omega_{-}$satisfy

$$
H \Omega_{+}=H \Omega_{-}=0
$$

and are called vacua. There exist dense domains $\mathscr{D}_{ \pm} \subset \mathscr{H}_{ \pm}$such that, for every unit vector $\psi_{ \pm} \in \mathscr{D}_{ \pm}$, one has that $\left\langle\psi_{ \pm}, \varphi(\mathbf{x}, t) \psi_{ \pm}\right\rangle$is a continuous function of $\mathbf{x}$ and $t$, with

$$
\lim _{|\mathbf{x}| \rightarrow \infty}\left\langle\psi_{ \pm}, \varphi(\mathbf{x}, t) \psi_{ \pm}\right\rangle= \pm \sqrt{\zeta}
$$

where $\zeta=\xi+O(1)$ is a positive constant. These results are proven in [5]. In [6] the spaces $\mathscr{H}_{s}$ and $\mathscr{H}_{s}$ have been constructed, and it is shown that

$$
\inf \operatorname{spec} H_{\mid \mathscr{H}_{s}}, \inf \operatorname{spec} H_{\mid \mathscr{H}_{3}}=O(1 / \lambda) \text {, }
$$

and that there are dense domains $\mathscr{D}_{s} \subset \mathscr{H}_{s}$ and $\mathscr{D}_{s} \subset \mathscr{H}_{s}$ such that, for every unit vector $\psi_{s} \in \mathscr{D}_{s},\left\langle\psi_{s}, \varphi(\mathbf{x}, t) \psi_{s}\right\rangle$ is continuous in $\mathbf{x}$ and $t$, with

$$
\lim _{\mathbf{x} \rightarrow \pm \infty}\left\langle\psi_{s}, \varphi(\mathbf{x}, t) \psi_{s}\right\rangle= \pm \sqrt{\zeta}
$$

while, for every $\psi_{3} \in \mathscr{D}_{3}$,

$$
\lim _{\mathbf{x} \rightarrow \pm \infty}\left\langle\psi_{3}, \varphi(\mathbf{x}, t) \psi_{s}\right\rangle=\mp \sqrt{\zeta} .
$$

As one might expect on the basis of Ehrenfest's theorem in quantum mechanics, the expectation values $\left\langle\psi_{ \pm}, \varphi(\mathbf{x}, t) \psi_{ \pm}\right\rangle$are close to classical solutions in $\Gamma_{ \pm}$, for $\lambda$ small, while $\left\langle\psi_{s}, \varphi(\mathbf{x}, t) \psi_{s}\right\rangle$ and $\left\langle\psi_{s}, \varphi(\mathbf{x}, t) \psi_{s}\right\rangle$ are close to solutions in $\Gamma_{s}, \Gamma_{s}$, respectively, for $\lambda$ small. See [7] for a discussion of the classical limit of quantum field theory.

The results sketched here are proven by making use of the Euclidean approach to quantum field theory [8]. The Euclidean approach is based on an analytic continuation of expectation values of products of field operators in the time variables from the real to the imaginary axis. This procedure is well known from non-relativistic Schrödinger quantum mechanics with a Hamiltonian of the form $H=-\Delta+V$, where $\Delta$ is the Laplacian and $V$ is a potential (multiplication operator). Then $e^{-t H}$ can be expressed in terms of Wiener integrals, with the help of the Feynman-Kac formula. By the remark following (1.8) and (1.9), the Hamiltonian of the $\lambda \varphi_{2}^{4}$-model is of the form $H=-\Delta+V$, where, formally, $\Delta$ is a Laplacian in infinitely many variables and $V=V(\varphi)$ is a multiplication operator. It is therefore tempting to consider expectation values of the following type: Let $x_{i}=\left(\mathbf{x}_{i}, t_{i}\right) \in \mathbb{R}^{2}$, for $i=1, \ldots, n$, with $t_{1}<t_{2}<\ldots<t_{n}$. We define the Euclidean Green functions by

$$
G_{ \pm}^{(n)}\left(x_{1}, \ldots, x_{n}\right)=\left\langle\Omega_{ \pm}, \varphi\left(\mathbf{x}_{1}\right) e^{-\left(t_{2}-t_{1}\right) H} \varphi\left(\mathbf{x}_{2}\right) e^{-\left(t_{3}-t_{2}\right) H} \ldots e^{-\left(t_{n}-t_{n-1}\right) H} \varphi\left(\mathbf{x}_{n}\right) \Omega_{ \pm}\right\rangle,
$$

where $\langle\cdot, \cdot\rangle$ denotes the scalar product on $\mathscr{H}_{ \pm}$. By making use of a Feynman-Kac formula for the operators $e^{-\left(t_{j+1}-t_{j}\right) H}$ which have norm $\leqq 1$, since $H \geqq 0$ and $t_{j+1}-t_{j} \geqq 0$, one can show $[4,8]$ that the distributions $G_{ \pm}^{(n)}\left(x_{1}, \ldots, x_{n}\right)$ are given as moments of some probability measure, $d \mu_{ \pm}(\phi)$, on Schwartz distribution space 
$\mathscr{S}_{\text {real }}^{\prime}\left(\mathbb{R}^{2}\right)$. In order to be more precise, we define the Euclidean action of the theory,

$$
S(\phi)=\frac{1}{2} \int_{\mathbb{R}^{2}}\left[:(\nabla \phi)^{2}(x):+\frac{\lambda}{2}:\left(\phi(x)^{2}-\xi\right)^{2}:\right] d^{2} x,
$$

where $\phi(x)$ is a random field over Euclidean space-time $\mathbb{R}^{2}$, with values in (a subspace of) $\mathscr{S}_{\text {real }}^{\prime}\left(\mathbb{R}^{2}\right)$, and the double colons now indicate Euclidean Wick- (also called Ito-) ordering.

Formally, the measures $d \mu_{ \pm}(\phi)$ are expressed by the Euclidean Gell-MannLow formula $[4,8]$,

$$
d \mu_{ \pm}(\phi)=\frac{1}{Z} e^{-S(\phi)} D \phi_{ \pm},
$$

where $D \phi_{ \pm}$is a formal Lebesgue measure on a subspace of $\mathscr{S}_{\text {real }}^{\prime}\left(\mathbb{R}^{2}\right)$ of distributions with boundary conditions

$$
\phi(x) \rightarrow \pm \sqrt{\zeta}, \text { as }|x| \rightarrow \infty
$$

and $Z$ is a (divergent) normalization factor, chosen such that

$$
\int_{\mathscr{S}_{\text {real }}^{\prime}\left(\mathbb{R}^{2}\right)} d \mu_{ \pm}(\phi)=1
$$

Formally, the Euclidean Green functions $G_{ \pm}^{(n)}$ are then given by

$$
G_{ \pm}^{(n)}\left(x_{1}, \ldots, x_{n}\right)=\int_{\mathscr{S}_{\text {real }}^{\prime}\left(\mathbb{R}^{2}\right)} \prod_{j=1}^{n} \phi\left(x_{j}\right) d \mu_{ \pm}(\phi) .
$$

In constructive field theory [4-9], one has succeeded in giving a mathematically precise meaning to the formal expressions (1.17)-(1.19). In particular, a formula like (1.19) has been proven as a rigorous theorem [5].

Note that the one-point Green function

$$
G_{ \pm}^{(1)}(x)=\int_{\mathscr{S}_{\text {real }}^{\prime}\left(\mathbb{R}^{2}\right)} \phi(x) d \mu_{ \pm}(\phi)=\left\langle\Omega_{ \pm}, \varphi(\mathbf{x}) \Omega_{ \pm}\right\rangle= \pm \sqrt{\zeta}= \pm \sqrt{\xi}+O(\sqrt{\lambda})
$$

corresponds to the minima $\varphi(\mathbf{x})= \pm \sqrt{\xi}$ of the classical Hamilton functional of $\lambda \varphi_{2}^{4}$-theory.

The Euclidean approach to constructing $\lambda \varphi_{2}^{4}$-theory in the soliton representations, i.e., to constructing the Hilbert spaces $\mathscr{H}_{s}$ and $\mathscr{H}_{s}$ and the dynamics on these spaces, is more recent $[10,11]$. The idea is to construct the Euclidean Green functions, $G_{s}^{(n)}\left(x_{1}, \ldots, x_{n}\right)$, of a quantum field $s(\mathbf{x}, t)=e^{i t H} s(\mathbf{x}) e^{-i t H}$, the soliton field, which has the following properties: $s(\mathbf{x}, t)$, smeared out with a testing function, maps the dense domains $\mathscr{D}_{ \pm} \subset \mathscr{H}_{ \pm}$to dense domains contained in $\mathscr{H}_{s}, \mathscr{H}_{s}$, respectively, and it maps the domains $\mathscr{D}_{s} \subset \mathscr{H}_{s}$ and $\mathscr{D}_{3} \subset \mathscr{H}_{s}$ to dense domains in $\mathscr{H}_{+}$ and $\mathscr{H}_{-}$respectively. The commutation relations between $s$ and $\varphi$ are given by

$$
s(\mathbf{x}, t) \varphi(\mathbf{y}, t)=\left\{\begin{array}{lll}
-\varphi(\mathbf{y}, t) s(\mathbf{x}, t), & \text { if } & \mathbf{x}<\mathbf{y} \\
\varphi(\mathbf{y}, t) s(\mathbf{x}, t), & \text { if } & \mathbf{x}>\mathbf{y},
\end{array}\right.
$$

(a similar formula holds for $s$ and $\pi$ ); see [6]. Defining $s(f):=\iint s(\mathbf{x}, t) f(\mathbf{x}, t) d \mathbf{x} d t$, where $f$ is a suitable testing function, one sees from (1.20), that the "soliton form 
factors" have the following behaviour:

$$
\lim _{\mathbf{x} \rightarrow \pm \infty}\left\langle s(f) \Omega_{+}, \varphi(\mathbf{x}, t) s(f) \Omega_{+}\right\rangle=-\lim _{\mathbf{x} \rightarrow \pm \infty}\left\langle s(f) \Omega_{-}, \varphi(\mathbf{x}, t) s(f) \Omega_{-}\right\rangle= \pm \sqrt{\zeta},
$$

i.e., $\left\langle s(f) \Omega_{ \pm}, \varphi(\mathbf{x}, t) s(f) \Omega_{ \pm}\right\rangle$is a function close to a solution of the classical field equation (1.1) in the space $\Gamma_{s}, \Gamma_{\bar{s}}$, respectively.

What has been accomplished in [11] is to find a mathematically precise formula for the Euclidean Green functions,

$$
G_{s}^{(n)}\left(x_{1}, \ldots, x_{n}\right)=\left\langle\Omega_{+}, s\left(\mathbf{x}_{1}\right) e^{-\left(t_{2}-t_{1}\right) H} s\left(\mathbf{x}_{2}\right) e^{-\left(t_{3}-t_{2}\right) H} \ldots e^{-\left(t_{n}-t_{n-1}\right) H} s\left(\mathbf{x}_{n}\right) \Omega_{+}\right\rangle,
$$

of the soliton field $s(\mathbf{x}, t)$, in terms of functional integrals. The formula found in [11] is obtained as follows: We define

$$
M_{\underline{x}}=\mathbb{R}^{2} \backslash\left\{x_{1}, \ldots, x_{m}\right\},
$$

where $\underline{x}=\left\{x_{1}, \ldots, x_{m}\right\}$ is an $m$-tuple of distinct points in $\mathbb{R}^{2}$. Obviously, $M_{\underline{x}}$ is not simply connected, and there exist, therefore, non-trivial, real line bundles, $E$, over $M_{\underline{x}}$ with fibre $\mathbb{R}$ and structure group $\mathbb{Z}_{2}$. Such bundles can be characterized completely by the holonomy of a flat connection:

With every $x_{i}$ we associate a number $q_{i}=0$, or 1 . If $\omega$ is a loop in $M_{x}$ we denote by $n\left(\omega, x_{i}\right)$ the winding number of $\omega$ with respect to $x_{i}$. We define the holonomy of a flat connection along $\omega$ by

$$
U(\omega)=(-1)^{\sum_{i=1}^{m} q_{i} n\left(\omega, x_{i}\right)}
$$

From now on, we set $m=2 n, q_{i}=1, \forall i$. Let $\nabla_{\underline{x}}$ be the covariant derivative acting on sections of $E$. We define a covariant action

$$
S_{\underline{x}}(\phi)=\int_{M_{\underline{x}}} \frac{1}{2}\left[:\left(\nabla_{\underline{x}} \phi\right)^{2}:(x)+\frac{\lambda}{2}:\left(\phi(x)^{2}-\xi\right)^{2}:\right] d^{2} x .
$$

Let $Z$ be as in (1.17), i.e.,

$$
Z=\int_{\mathscr{S}_{\text {real }}} e^{-S(\phi)} D \phi_{ \pm}
$$

We define the Euclidean Green functions of the field $s(x)$ by

$$
G_{s}^{(2 n)}\left(x_{1}, \ldots, x_{2 n}\right)=\left[\frac{1}{Z} \int_{\Gamma(E)} e^{-S_{\underline{x}}(\phi)} D_{E} \phi_{+}\right]_{\mathrm{ren} .} .
$$

Here $\Gamma(E)$ is a space of section distributions of $E, D_{E} \phi_{+}$is a formal Lebesgue measure on $\Gamma(E)$ with boundary condition as in $(1.18)$, and $[(\cdot)]_{\text {ren. }}$ indicates a multiplicative renormalization.

It has been shown in [11] how to give rigorous mathematical meaning to (1.26): Let $d \mu_{0}(\phi)$ be the Gaussian measure with mean 0 and covariance

$$
\left(-\Delta+m^{2}\right)^{-1}, m^{2}=2 \lambda \xi,
$$

and $d \mu_{\underline{x}}(\phi)$ the Gaussian on $\Gamma(E)$ with mean 0 and covariance

$$
\left(-\Delta_{\underline{x}}+m^{2}\right)^{-1},
$$


where $\Delta_{\underline{x}}$ is the covariant Laplacian acting on sections of $E$, with $\underline{x}=\left\{x_{1}, \ldots, x_{2 n}\right\}$ and $q_{i}=1, i=1, \ldots, 2 n$. Let

$$
V_{+}(\phi)=\int\left[\frac{\lambda}{2}:\left(\phi(x)^{2}-\xi\right)^{2}:-\frac{m^{2}}{2}: \phi(x)^{2}:\right] d^{2} x
$$

with boundary condition $\phi(x) \rightarrow+\sqrt{\xi}$ at $\infty$. Then

$$
G_{s}^{(2 n)}\left(x_{1}, \ldots, x_{2 n}\right)=\frac{z(\underline{x})}{\tilde{Z}} \int e^{-V+(\phi)} d \mu_{\underline{x}}(\phi),
$$

where $\tilde{Z}=\int e^{-V+(\phi)} d \mu_{0}(\phi)$, and

$$
z(\underline{x})=\operatorname{det}\left(\left(-\Delta_{\underline{x}}+m^{2}\right) /\left(-\Delta+m^{2}\right)\right)_{\text {ren. }}^{-1 / 2} .
$$

The subscript "ren." on the right of (1.28) indicates a multiplicative renormalization, [11]. It is reasonably straightforward to make sense of (1.27) and (1.28), [11].

Physicists cannot stop at the point where they have rigorously interpreted their objects of interest. They want to calculate them explicitly. Hence we set out to, at least approximately, calculate (1.26). One standard scheme to accomplish this is the semi-classical expansion.

We multiply $S(\phi)$ and $S_{\underline{x}}(\phi)$ by $\frac{1}{\hbar}, \mathbf{h}=$ Planck's quantum of action, and try to calculate (1.26) for small $\boldsymbol{\hbar}$. By rescaling the field $\phi: \phi \rightarrow \sqrt{\hbar} \phi$, an expansion in powers of $\sqrt{\hbar}$ turns out to be closely related to an expansion in powers of $\sqrt{\lambda}$ (for $\boldsymbol{h}=1$ ).

The term of order $\left(\frac{1}{\hbar}\right)$ is classical: It is obtained by minimizing the actions $S(\phi)$ and $S_{\underline{x}}(\phi)$. While this is trivial for $S(\phi)$, it is not completely standard for $S_{\underline{x}}(\phi)$. This variational problem and generalizations thereof form the subject matter of this paper.

The term of order 1 (one-loop correction) is given by a quotient of determinants:

Let $\tilde{\phi}$ be the minimizer of $S_{\underline{x}}(\phi)$. Set $\phi=\tilde{\phi}+\sqrt{\mathfrak{h}} \psi$ and expand $S_{\underline{x}}(\phi)$ to second order in the "variation" $\psi$. Then

$$
S_{\underline{x}}(\phi)=S_{\underline{x}}(\tilde{\phi})+\frac{\hbar}{2}\left\langle\psi, Q_{\tilde{\phi}} \psi\right\rangle,
$$

where $Q_{\tilde{\phi}}$ is a non-negative quadratic form. Similarly, setting $\phi= \pm \sqrt{\xi}+\sqrt{\hbar} \psi$, we find

$$
S(\phi)=0+\frac{\boldsymbol{h}}{2}\langle\psi, Q \psi\rangle
$$

where $Q=-\Delta+m^{2}, m^{2}=2 \lambda \xi$. Hence the 0 -order term is given by

$$
\operatorname{det}\left(Q_{\tilde{\phi}} /\left(-\Delta+m^{2}\right)\right)_{\text {ren. }}^{-1 / 2},
$$

where "ren." indicates a multiplicative renormalization. Terms of order $\left(\boldsymbol{\hbar}^{n / 2}\right), n \geqq 1$, can be computed in terms of Feynman diagrams; [with propagators given by $\left.Q_{\tilde{\phi}}^{-1},\left(-\Delta+m^{2}\right)^{-1}\right]$. 
From these considerations we extract two important mathematical problems:

I. Establish existence, uniqueness and regularity of the minimizer, $\tilde{\phi}$, for $S_{\underline{x}}(\phi)$. II. Analyse the spectral properties of $Q_{\tilde{\phi}}$; in particular, establish that $0 \notin \sigma\left(Q_{\tilde{\phi}}\right)$ and calculate the determinant in (1.29).

The solution of problems I and II will reveal that, to order $\hbar^{0}$,

$$
G_{s}^{(2)}\left(x_{1}, x_{2}\right) \sim a\left|x_{1}-x_{2}\right|^{-1 / 2} \exp \left[-b \boldsymbol{h}^{-1}\left|x_{1}-x_{2}\right|\right],
$$

as $\left|x_{1}-x_{2}\right| \rightarrow \infty$, where $a$ and $b$ are calculable from $\tilde{\phi}$. Our results in Sects. 2-5 imply that $b=\frac{2 \sqrt{2}}{3} \xi \sqrt{\lambda \xi}+O(\mathbf{h})$.

Remark. The solution of analogous problems for the Green functions $G_{ \pm}^{(n)}\left(x_{1}, \ldots, x_{n}\right)$ defined in (1.19) can be inferred from [5].

Example 2. [U(1)-Higgs model in three space-time dimensions.] We consider a gauge field model with a Lagrange density, $\mathscr{L}$, given by

$$
\mathscr{L}(\varphi, A)=\frac{1}{2 e^{2}}(d A)^{2}+\frac{1}{2}|(d-i A) \varphi|^{2}-V(|\varphi|),
$$

where $A=\left(A_{\mu} d x^{\mu}\right)$ is a $U(1)$-connection (gauge field) on a complex line bundle over three-dimensional Minkowski space, and $\varphi$ (the Higgs field) denotes a section of this bundle. The symbol " $d$ " denotes an exterior derivative, and $d-i A$ denotes the covariant derivative. Finally, $V(|\varphi|)$ (the Higgs potential) is a polynomial in $|\varphi|$ which is bounded from below; typically

$$
V(|\varphi|)=\frac{\lambda}{2}\left(|\varphi|^{2}-\xi\right)^{2}, \quad \lambda>0
$$

The energy of a configuration $(\varphi, A)$ at some fixed time $t$ is given by

$$
\begin{aligned}
H(\varphi, \mathbf{A})= & \int\left[\frac{1}{2 e^{2}}\left(\mathbf{E}^{2}+B^{2}\right)(\mathbf{x}, t)+\frac{1}{2}\left|\left(\partial_{t} \varphi\right)(\mathbf{x}, t)\right|^{2}\right. \\
& \left.+\frac{1}{2}|(\nabla-i \mathbf{A}) \varphi(\mathbf{x}, t)|^{2}+V(|\varphi(\mathbf{x}, t)|)\right] d^{2} \mathbf{x},
\end{aligned}
$$

where we have chosen the temporal gauge $A_{0}(\mathbf{x}, t) \equiv 0,\left(A_{0}\right.$ is the component of $A$ in the time direction), $\mathbf{E}:=\partial_{t} \mathbf{A}$, with $\mathbf{A}=\left(A_{1}, A_{2}\right)$, and $B:=\nabla \wedge \mathbf{A}, \nabla:=\left(\partial_{1}, \partial_{2}\right)$; note that, in two space dimensions, $B$ is a scalar.

It is expected [12] that smooth field configurations $(\varphi, \mathbf{A})$ of finite energy, i.e., with $H(\varphi, \mathbf{A})<\infty$, and with a suitable gauge condition imposed on $\mathbf{A}$, define homotopy classes in $\pi_{1}\left(S^{1}\right)=\mathbb{Z}$. The homotopy class of a configuration $(\varphi, \mathbf{A})$ is given by the winding number of the map

$$
\left.\frac{\varphi(\mathbf{x}, t)}{|\varphi(\mathbf{x}, t)|}\right|_{|\mathbf{x}|=R}: S^{1} \rightarrow S^{1},
$$

as $R \rightarrow \infty$. It coincides with the gauge-invariant quantity 


$$
\frac{1}{2 \pi} \int B(\mathbf{x}, t) d^{2} \mathbf{x} \in \mathbb{Z}
$$

which is called the vorticity of $\mathbf{A}$.

Thus the space of smooth field configurations $(\varphi, \mathbf{A})$ of finite energy very likely decomposes into infinitely many topologically distinct classes labelled by an integer, their vorticity.

Static solutions of the classical Euler-Lagrange equations derived from (1.30) with vorticity \pm 1 have been exhibited in [12]. They are called vortex- and antivortex solutions. For the solution with vorticity $1, B(\mathbf{x})>0$ has a maximum at some point $\mathbf{x}_{0} \in \mathbb{R}^{2}, B(\mathbf{x})$ decreases exponentially fast in $\left|\mathbf{x}-\mathbf{x}_{0}\right|$, and $\int_{\mathbb{R}^{2}} B(\mathbf{x}) d^{2} \mathbf{x}=2 \pi$. Moreover, $|\varphi(\mathbf{x})|$ has a zero at $\mathbf{x}_{0},|\varphi(\mathbf{x})|$ approaches 1 exponentially fast in $\left|\mathbf{x}-\mathbf{x}_{0}\right|$, and $\arg \varphi(\mathbf{x}) \simeq \arctan \left(y-y_{0} / x-x_{0}\right)$, for $\left|\mathbf{x}-\mathbf{x}_{0}\right|$ large; (here $x$ is the 1-coordinate and $y$ the 2-coordinate of $\mathbf{x}$ ).

One may wonder what becomes of these classical solutions (or, more generally, of the different homotopy classes of finite-energy configurations of classical field theory) in quantum theory? The answer is similar to the one we have outlined above for the kinks of $\lambda \varphi_{2}^{4}$-theory. One attempts to construct a local quantum field, $v(\mathbf{x}, t)$, (the vortex field), such that the expectation values

$$
\langle v, B(\mathbf{x}, t) v\rangle,\langle v, \varphi(\mathbf{x}, t) v\rangle,
$$

where $v:=v(f) \Omega, v(f)=\iint v(\mathbf{x}, t) f(\mathbf{x}, t) d^{2} \mathbf{x} d t, f$ is a suitable testing function, and $\Omega$ is the vacuum vector of the quantum theory, resemble the solutions of vorticity 1 of the classical Euler-Lagrange equations which we have described above.

Again, it turns out that the most efficient method to construct the vortex field $v(\mathbf{x}, t)$ and the entire quantum theory is to use Euclidean methods [13]. One attempts to construct the Euclidean Green functions of the field $v(\mathbf{x}, t)$ and of its adjoint $v^{+}(\mathbf{x}, t)$. The Euclidean action of the $U(1)$-Higgs model is given by

$$
S(\phi, A)=\int_{\mathbb{R}^{3}}\left[\frac{1}{2 e^{2}}:(d A)^{2}(x):+:|(d-i A) \phi(x)|^{2}:+: V(|\phi(x)|):+ \text { c.t. }\right] d^{3} x .
$$

In (1.34), $\phi$ is a complex-valued random field on Euclidean space-time $\mathbb{R}^{3}$ (the Euclidean Higgs field), and $A$ is a random connection (the Euclidean gauge field); the double colons indicate Wick- (or Ito-) ordering, and "c.t." stands for a " $\phi$-mass counterterm" and for a constant counterterm (with coefficients that diverge, as a smoothing cutoff is removed). The counterterms in the definition of $S(\phi, A)$ turn out to be necessary to make the theory mathematically well defined [14].

We now sketch the construction of the Euclidean Green functions of the vortex fields $v(\mathbf{x}, t)$ and $v^{+}(\mathbf{x}, t)$, following [13]. We replace Euclidean space-time $\mathbb{R}^{3}$ by

$$
M_{\underline{x}}:=\mathbb{R}^{3} \backslash\left\{x_{1}, \ldots, x_{n}\right\},
$$

with $\underline{x}=\left\{x_{1}, \ldots, x_{n}\right\}$ a set of $n$ distinct points in $\mathbb{R}^{3}$. U(1)-bundles over some manifold $M$ are classified by the second cohomology group $H^{2}(M, \mathbb{Z})$. For $M=M_{\underline{x}}$,

$$
H^{2}\left(M_{x}, \mathbb{Z}\right)=\mathbb{Z} \oplus \mathbb{Z} \oplus \ldots \oplus \mathbb{Z} \quad \text { ( } n \text { summands) }
$$


The $n$ integers, $m_{1}, \ldots, m_{n}$, labelling an element of $H^{2}\left(M_{\underline{x}}, \mathbb{Z}\right)$ can be interpreted as magnetic charges of $n$ magnetic (Dirac) monopoles located at the points $x_{1}, \ldots, x_{n}$ of $\mathbb{R}^{3}$.

Let $A_{0}$ be a connection on a $U(1)$-bundle over $M_{\underline{x}}$ with magnetic charges $m_{1}, \ldots, m_{n}$, i.e.,

$$
\frac{1}{2 \pi} \int_{\Sigma} d A_{o}=\sum_{i: x_{i} \in \operatorname{In}(\Sigma)} m_{i},
$$

where $\Sigma$ is any closed surface in $\mathbb{R}^{3}$ enclosing some of the points $x_{1}, \ldots, x_{n}$ in its interior, $\operatorname{Int}(\Sigma)$. Every other connection on such a $U(1)$-bundle is of the form

$$
\tilde{A}=A_{o}+A,
$$

where $A$ is a globally defined 1 -form. One can choose $A_{o} \equiv A_{o}^{h}$ to be harmonic on $M_{\underline{x}}$. Hence its field strength, $F_{o}^{h}$, is given by

$$
F_{o}^{h}(\underline{x}, \underline{m})=2 \pi \sum_{i} m_{i} * d \Delta^{-1} \delta_{x_{i}}
$$

(It is convenient to choose $A_{o}$ to be harmonic at least in small neighbourhoods of the points $x_{1}, \ldots, x_{n}$.)

We now define a modified Euclidean action

$$
S_{\underline{m}, \underline{x}}(\phi, A)=\int_{M_{\underline{x}}}\left\{\frac{1}{2 e^{2}}:\left(F_{o}+d A\right)^{2}(x):+\frac{1}{2}:\left|\left(\nabla_{A+A_{o}} \phi\right)(x)\right|^{2}:+: V(|\phi|)(x):+ \text { c.t. }\right\} d^{3} x,
$$

where $\nabla_{A+A_{o}}$ is the covariant derivative on the associated complex line bundle. Suppose now that $x \equiv\left(x_{1}, \ldots, x_{n}\right)=\left(y_{1}, \ldots, y_{k}, z_{1}, \ldots, z_{k}\right), m_{y_{j}}=1, m_{z_{j}}=-1$. Then the Green functions of $v(y), v^{+}(z)$ are given by

$$
G\left(y_{1}, \ldots, y_{k}, z_{1}, \ldots, z_{k}\right)=\left[\frac{1}{Z} \int e^{-S_{\underline{m}, \underline{x}}(\phi, A)} D A D \phi\right]_{\mathrm{ren} .} .
$$

See [13] for a more precise definition of the right-hand side of (1.39).

It is of interest to attempt to evaluate (1.39) within a semi-classical expansion. The leading term of order $\frac{1}{\hbar}$ is again obtained by minimizing $S_{\underline{m}, \underline{x}}(\phi, A)$. This variational problem is not well posed, for two reasons:

(a) Gauge-invariance; $\left(|d \tilde{A}|^{2}\right.$ is not coercive).

(b) $S_{\underline{m}, \underline{x}}(\phi, A)$ is ill-defined (divergent).

Difficulty (b) is resolved as follows. We assume that $\sum_{j=1}^{n} m_{j}=0$, (neutrality). Let $\Omega$ be a ball containing $x_{1}, \ldots, x_{n}$, and $\Omega_{1}=\{x: \operatorname{dist}(x, \Omega) \leqq 1\}$. We choose $A_{o}$ such that $F_{o}$ is harmonic in $\Omega$ and vanishes outside $\Omega_{1}$. With this choice of $A_{o}$, $\frac{1}{2 e^{2}} \int F_{o}^{2}(x) d^{3} x$ diverges near $x_{1}, \ldots, x_{n}$. The divergence is universal and can be removed by replacing $\frac{1}{2 e^{2}} \int F_{o}^{2}(x) d^{3} x$ by

$$
\frac{4 \pi}{e^{2}} \sum_{i<j} \frac{m_{i} m_{j}}{\left|x_{i}-x_{j}\right|}+\frac{1}{2 e^{2}} \int_{\Omega^{c}}\left[F_{o}^{2}(x)-\left(F_{o}^{h}\right)^{2}(x)\right] d^{3} x,
$$


where $F_{o}^{h}$ is given by (1.37). That (1.40) is the right choice is well known from classical electromagnetism. With these choices the action functional is well defined on a space of Sobolev sections $\phi$ and connections $\tilde{A}$ of the bundle characterized by $\underline{m}$ and $\underline{x}$.

Difficulty (a) is removed as follows. If $\nabla A \in L^{2}\left(\mathbb{R}^{3}\right)$ then we can choose a gauge such that $\delta A=0$. Hence we replace $(d A)^{2}$ by $(\nabla A)^{2}$ in the definition of $S_{\underline{m}, \underline{x}}$. Moreover, we require that

$$
\int_{K} A d^{3} x=0,
$$

for some compact set $K$, e.g. $K=\Omega_{1}$, or $K=\Omega_{1} \backslash \Omega$.

From our discussion it follows that we should replace $S_{\underline{m}, \underline{x}}(\phi, A)$ by a functional $\tilde{S}_{\underline{m}, \underline{x}}(\phi, A)$ given by

$$
\begin{aligned}
& \widetilde{S}_{\underline{\underline{m}}, \underline{x}}(\phi, A)=\frac{4 \pi}{e^{2}} \sum_{i<j} \frac{m_{i} m_{j}}{\left|x_{i}-x_{j}\right|}+\frac{1}{2 e^{2}} \int_{\Omega^{c}}\left[F_{o}^{2}(x)-\left(F_{o}^{h}\right)^{2}(x)\right] d^{3} x \\
& \quad+\int_{M_{\underline{x}}}\left[\frac{1}{2 e^{2}}(\nabla A)^{2}(x)+2\left(A \delta F_{o}\right)(x)+\frac{1}{2}\left|\nabla_{A_{o}} \phi-i A \phi\right|^{2}(x)+V(|\phi(x)|)\right] d^{3} x .
\end{aligned}
$$

We have used here that $\int_{M_{x}} d A F_{o} d^{3} x=\int_{M_{x}} A \delta F_{o} d^{3} x+$ boundary terms. The boundary terms turn out to vanish. The following mathematical problems arise:

III. The first step in setting up a semi-classical expansion is to minimize

$$
\widetilde{S}_{\underline{m}, \underline{x}}(\phi, A)
$$

for fixed $\underline{m}, \underline{x}$, under the conditions that $\sum_{j=1}^{n} m_{j}=0$ and $\int_{\mathbf{K}} A d^{3} x=0$ and to prove regularity and other properties of the minimizer.

IV. The second step is to control the spectrum of the fluctuation operator (the $2^{\text {nd }}$ variation of $\widetilde{S}_{m, x}$ ) around the minimizer.

This problem is not only of interest in quantum field theory, but also in the study of Abrikosov vortices in Type II superconductors [15].

The techniques developed in Sect. 2, 3, and 5 of this paper can be used to study problems III and IV. Since we are aiming at a rather detailed understanding of the properties of minimizers which requires a considerable amount of work, our results will appear in a separate article with $U$. Studer.

Next, we describe some problems in the statistical physics of thermal equilibrium which lead to variational problems on vector bundles. As mentioned at the beginning, the problems we propose to study are connected with the energetics of topological defects in ordered media [1] and their statistical properties at very low temperature. An example of such a problem is the following one.

Example 3. (Landau-Ginzburg theory of Bloch walls.) Consider a highly anisotropic ferromagnet with order parameter (spin field) $\varphi(x) \in \mathbb{R}$ in three dimensions. We make a standard Landau-Ginzburg ansatz for the energy of a configuration $\varphi$ :

$$
E(\varphi)=\int\left[\frac{1}{2}(\nabla \varphi)^{2}(x)+\frac{\lambda}{2}\left(\varphi(x)^{2}-1\right)^{2}\right] d^{3} x .
$$


We propose to study the shape of Bloch walls separating regions of different magnetization, $\varphi$, from each other. The simplest way to do that is to choose some smooth, closed (possibly knotted) curves, $\mathscr{L}=\left(\mathscr{L}_{1}, \ldots, \mathscr{L}_{k}\right)$ and to require that they be the boundary curves of a system of Bloch walls. This is done as follows. We define

$$
M_{\mathscr{L}} \equiv \mathbb{R}^{3} \backslash \mathscr{L}
$$

and study real line bundles over $M_{\mathscr{L}}$ with structure group $\mathbb{Z}_{2}$. These bundles are uniquely characterized by numbers $q_{1}, \ldots, q_{k}$, with $q_{i}=0$, or 1 , for all $i$. If $\omega$ is a loop in $M_{\mathscr{L}}$ one requires the holonomy condition

$$
U(\omega)=(-1)^{\sum_{i=1}^{k} q_{i} n\left(\omega, \mathscr{L}_{i}\right)},
$$

where $n\left(\omega, \mathscr{L}_{i}\right)$ is the winding number of $\omega$ with $\mathscr{L}_{i}$. There is a flat connection over $M_{\mathscr{L}}$ with holonomy given by (1.45). The idea is now to minimize $E(\varphi)$, given in (1.43), on a space of sections of a bundle over $M_{\mathscr{L}}$ whose holonomy is given by (1.45), with $q_{1}=\ldots=q_{k}=1$. It is easy to see that any section of such a bundle must vanish on a "surface" bounded by $\mathscr{L}$. The surface where the minimizer, $\varphi_{o}$, of $E(\varphi)$ vanishes gives a description of the geometry of a Bloch wall bounded by $\mathscr{L}$ at low temperatures. One of our goals is to understand the relation between this problem and standard minimal surface theory. Corrections to the classical theory, valid at zero temperature, are provided by the low temperature expansion which is analogous to the semi-classical expansion in Examples 1 and 2.

Remark. Conventionally, one follows a different approach. One confines the system to a bounded region $\Lambda$ (a ball or cube) and requires that $\mathscr{L} \subset \partial \Lambda$. One then chooses boundary conditions, $\varphi(x)= \pm 1$, in the connected regions of $\partial \Lambda \backslash \mathscr{L}$ which force Bloch walls through the system in $\Lambda$ that are bounded by $\mathscr{L}$. Our approach is somewhat different, but gives an equivalent description of the geometry of Bloch walls when the diameter of the curves $\mathscr{L}_{i}, i=1, \ldots, k$, becomes large. Our approach is of interest in connection with Ising spin glasses and with $\mathbb{Z}_{2}$-gauge theory which is dual to the theory of Ising ferromagnets, in the sense of Kramers-Wannier duality.

There are further interesting problems closely related to the one just discussed. As an example, consider an $n$-states "Potts model," with $n \geqq 3$. It describes an ordered medium with an order parameter $\varphi(x) \in \mathbb{C}$ which preferably takes a value close to an $n^{\text {th }}$ root of unity. The Landau-Ginzburg ansatz for the energy of a configuration $\varphi$ is

$$
E(\varphi)=\int\left[\frac{1}{2}|\nabla \varphi(x)|^{2}+V(\varphi(x))\right] d^{3} x,
$$

where $V$ is a potential which is invariant under

$$
\varphi \mapsto e^{\frac{2 \pi i l}{n}} \varphi
$$

for $l=1, \ldots, n-1$, and has deep minima at

$$
\varphi=e^{\frac{2 \pi i l}{n}}, \quad l=0,1, \ldots, n-1 .
$$


To study Bloch walls in the Potts model we choose curves $\mathscr{L}=\left(\mathscr{L}_{1}, \ldots, \mathscr{L}_{k}\right)$, set $M_{\mathscr{L}}=\mathbb{R}^{3} \backslash \mathscr{L}$ and impose holonomy conditions

$$
U(\omega)=e^{\frac{2 \pi i}{n}} \sum_{j=1}^{k} l_{j} n\left(\omega, \mathscr{L}_{j}\right),
$$

where each $l_{j}=1, \ldots, n-1$, for $j=1, \ldots, k$. We must minimize $E(\varphi)$ over a space of sections, $\varphi$, with prescribed holonomy (1.49).

Now, every section $\varphi$ of the bundle specified by (1.49) determines branched "surfaces" defined as the set of those $x$ where $\arg \varphi(x)$ is in the middle between the arguments of two $n^{\text {th }}$ roots of unity. These surfaces are bounded by $\mathscr{L}$ and give a description of the geometry of Bloch walls bounded by $\mathscr{L}$.

Example 4. (Point defects in a three-dimensional Heisenberg ferromagnet.) Consider a three-dimensional ferromagnet with order parameter $\varphi \in \mathbb{R}^{3}$ and energy functional

$$
E(\varphi)=\int\left[\frac{1}{2}|(\nabla \varphi)(x)|^{2}+V(|\varphi(x)|)\right] d^{3} x .
$$

It is assumed that $V$ has a deep minimum on $|\varphi|=1$, e.g.

$$
V(|\varphi|)=\frac{\lambda}{2}\left(|\varphi|^{2}-1\right)^{2}
$$

Now, we remove $n$ points $\underline{x}=\left\{x_{1}, \ldots, x_{n}\right\}$ from $\mathbb{R}^{3}$, set $M_{\underline{x}}=\mathbb{R}^{3} \backslash \underline{x}$, and assign integers $m_{1}, \ldots, m_{n}$ to $x_{1}, \ldots, x_{n}$. Let $\Sigma_{p}$ be any closed surface in $\mathbb{R}^{3}$ enclosing the points $x_{p(1)}, \ldots, x_{p(l)}, l \leqq n$. We require that the degree ( $2^{\text {nd }}$ homotopy class) of the map

be given by

$$
\left.\frac{\varphi(x)}{|\varphi(x)|}\right|_{x \in \Sigma_{p}}: \Sigma_{p} \rightarrow S^{2}
$$

$$
\sum_{j=1}^{l} m_{p(j)} \in \mathbb{Z},
$$

for every $\Sigma_{p}$ with the properties specified above.

The problem is to minimize $E(\varphi)$ subject to the constraints (1.51), (1.52). This problem is related, mathematically, to the $U(1)$-Higgs problem described earlier. Other variants of this problem concern systems in finite regions, $\Lambda$, with topologically non-trivial boundary conditions on $\partial \Lambda$. For example, one may restrict the order parameter $\varphi$ of the model considered above to a ball, $K_{R}$, of radius $R$, centered at 0 , and minimize $E(\varphi)$, defined in (1.50), under the condition that

$$
\left.\frac{\varphi(x)}{|\varphi(x)|}\right|_{x \in \partial K_{R}}: S^{2} \rightarrow S^{2}
$$

has a prescribed degree $n$. One would like to estimate the number and approximate positions of zeros of $\varphi(x)$ in $K_{R}$. A very similar problem has been studied in $[16,17]$. A related problem in four dimensions is encountered in the analysis of Skyrmions in an $S U(2)$ non-linear sigma-model or in a "linear" version of this theory. 


\section{General Existence Results}

Examples 1 and 3 of Sect. 1 suggest to consider the following model problem:

Let $M$ be an $n$-dimensional Riemannian manifold countable at infinite - for example, $\mathbb{R}^{\mathbf{3}} \backslash \mathscr{L}$, where $\mathscr{L}$ is compact - with non-trivial, countable fundamental group $\pi_{1}(M)$. Consider a vector bundle $E$ over $M$ with fibre $F \cong \mathbb{R}^{k}$ or $\mathbb{C}^{k}$ and structure group $G \subset S O(k)$ or $S U(k)$ acting on $F$, and a flat connection on $E$. The holonomy operators of the flat connection on $E$ define a representation of $\pi_{1}(M)$ with values in $G$ as follows: With each loop $\omega \subset M$ representing an element of $\pi_{1}(M)$ we can associate a holonomy operator $g_{\omega} \in G$, and $g_{\omega}$ only depends on the homotopy class $[\omega] \in \pi_{1}(M)$ of $\omega$.

Denote by $\tilde{M}$ the universal cover of $M$. Then sections of $E$ can be viewed as mappings $\varphi: \tilde{M} \rightarrow F$, satisfying the condition

$$
\varphi \circ[\omega]=g_{[\omega]} \varphi, \text { for all }[\omega] \in \pi_{1}(M),
$$

where we think of $\pi_{1}(M)$ as acting through covering transformations on $\tilde{M}$.

For $\Omega \subset \tilde{M}, 1 \leqq p<\infty$, we define

$$
L^{p}(\Omega ; F)=\left\{\varphi: \Omega \rightarrow F ; \int_{\Omega}|\varphi|^{p} d x<\infty\right\}
$$

where $|\cdot|$ is the norm induced by the scalar product $\langle\cdot, \cdot\rangle$ on $F$, and $\int_{\Omega} \ldots$ denotes integration with respect to the volume form on $\tilde{M}$. We say that $\varphi \in L_{\text {loc }}^{p}(\tilde{M} ; F)$ if $\varphi \in L^{p}(\Omega ; F)$, for all $\Omega$ compactly contained in $\tilde{M}(\Omega \Subset \tilde{M})$. Also let $\nabla$ denote ordinary (componentwise) differentiation of functions $\varphi: \tilde{M} \rightarrow F$. Then for any function $\varphi \in L_{\text {loc }}^{1}(\tilde{M} ; F)$ we can define the distributional derivative $\nabla \varphi$ by restricting to local coordinate charts $\Omega$. The action of $\nabla \varphi$ on smooth functions with support in $\Omega$ is interpreted as usual via integration by parts. Analogously, we can define $\nabla^{k} \varphi$ $=\frac{\partial^{|k|}}{\partial x_{1}^{k_{1}} \ldots \partial x_{n}^{k_{n}}} \varphi$ for any multi-index $k=\left(k_{1}, \ldots, k_{n}\right),|k|=k_{1}+\ldots+k_{n} \in \mathbb{N}_{o}$. In this way we obtain the Sobolev spaces

$$
H^{m, p}(\Omega ; F)=\left\{\varphi \in L^{p}(\Omega ; F) ; \nabla^{k} \varphi \in L^{p}(\Omega ; F), 0 \leqq|k| \leqq m\right\}
$$

with norm

$$
\|\varphi\|_{H^{m, p}}=\sum_{0 \leqq|k| \leqq m}\left(\int_{\Omega}\left|\nabla^{k} \varphi\right|^{p} d x\right)^{1 / p} .
$$

Note that $H^{o, p}=L^{p}$. Moreover, we write $H_{\text {loc }}^{m, p}(\tilde{M} ; F)=\bigcap_{\Omega \in \tilde{M}} H^{m, p}(\Omega ; F)$, as before.

The big advantage of working in Sobolev spaces rather than in spaces of continuously differentiable functions is that Sobolev-spaces are complete with respect to integral norms related to the action integrals of the problems we are considering - which is not the case for spaces of classically differentiable functions. Computations, however, can almost always be performed as if we were working with smooth functions, due to the fact that (for smooth domains $\Omega$ ) smooth functions are dense in $H^{m, p}(\Omega)$, for all $m \in \mathbb{N}_{o}, 1 \leqq p<\infty$, see Adams [18]. Moreover, we have the Rellich compactness theorem and the Sobolev embedding theorem at our disposal: 
Theorem. Let $\Omega \subset \tilde{M}$ be smooth and bounded, $m \geqq 1,1 \leqq p<\infty, m p<n$. Then $H^{m, p}(\Omega) \hookrightarrow L^{q}(\Omega)$ continuously for any $q \in\left[1, \frac{n p}{n-m p}\right]$. The embedding $H^{m, p}(\Omega) \hookrightarrow L^{q}(\Omega)$ is compact for any $q<\frac{n p}{n-m p}$. If $m p>n$, moreover, we have $H^{m, p}(\Omega) \hookrightarrow C^{k, \alpha}\left(\Omega_{1}\right)$, where $k \in \mathbb{N}_{0}, 0<\alpha<1$ and $0<\alpha \leqq m-k-\frac{n}{p} \leqq 1$. (Confer e.g.
Adams [18].)

The number $p^{*}=\frac{n p}{n-m p}>p$ is called the Sobolev exponent for the embedding of $H^{n, p}$. Actually, we have

$$
\|\varphi\|_{L^{p^{*}}} \leqq C\|\varphi\|_{H^{m, p}},
$$

with a constant $C$ independent of $\Omega$, and the remaining embedding estimates are derived from Hölder's inequality.

Of special importance in the following will be the spaces $H^{1,2}(\Omega ; F)$ which are separable Hilbert spaces with scalar product

$$
\langle\varphi, \psi\rangle_{H^{1,2}}=\int_{\Omega}(\langle\varphi, \psi\rangle+\langle\nabla \varphi, \nabla \psi\rangle) d x
$$

inducing the norm $\|\cdot\|_{H^{1,2}}$. In particular, we will be concerned with the special set of functions in $H_{\text {loc }}^{1,2}(\tilde{M} ; F)$ obeying the holonomy condition (2.1) and having finite energy.

For this, given a real-valued, $G$-invariant "potential" $V: F \rightarrow \mathbb{R}, \varphi \in H_{\text {loc }}^{1,2}(\tilde{M} ; F)$, and $\Omega \Subset \tilde{M}$ let

$$
E(\varphi ; \Omega)=\int_{\Omega}\left(|\nabla \varphi|^{2}+V(\varphi)\right) d x
$$

be the energy of $\varphi$ on $\Omega$. Observe that $E$ is $G$-invariant in the sense that

$$
E(g \varphi ; \Omega)=E(\varphi ; \Omega), \text { for all } g \in G
$$

and any $\varphi \in H_{\text {loc }}^{1,2}(\tilde{M} ; F), \Omega \Subset \tilde{M}$. Thus, if $\varphi$ satisfies (2.1), it makes sense to define

$$
E(\varphi)=\int_{M}\left(|\nabla \varphi|^{2}+V(\varphi)\right) d x=E(\varphi ; M)
$$

as the total energy of $\varphi$. [Note that we may regard $M$ as a fundamental domain in $\tilde{M}$, with respect to the action of the - in general infinite - group $\pi_{1}(M)$.]

The existence of a minimizer of $E$ satisfying (2.1) in a weak sense can be established under very general hypotheses concerning the potential $V$.

Theorem 2.1. Suppose $V: F \rightarrow \mathbb{R}$ is continuous, non-negative and coercive in the sense that

$\left(1^{\circ}\right) V(\varphi) \geqq C^{-1}|\varphi|^{2}-C$, for some constant $C>0$ and all $\varphi \in F$.

Also suppose that there exists a $C^{1}$-function $\varphi_{o}: \tilde{M} \rightarrow F$ satisfying (2.1) and such that $E\left(\varphi_{0}\right)<\infty$. Then there exists a function $\varphi \in H_{\text {loc }}^{1,2}(\tilde{M} ; F)$ satisfying (2.1) almost everywhere and minimizing $E$ in this class.

Proof. Let $\left(\Omega_{k}\right)$ be a sequence of compact domains $\Omega_{k} \Subset M$ exhausting $M$ and let $\mathscr{H}=\mathscr{H}^{(1,2)}$ be the space

$$
\mathscr{H}=\mathscr{H}^{(1,2)}=\left\{\varphi \in H_{\text {loc }}^{1,2}(\tilde{M} ; F) ; \varphi \text { satisfies }(2.1)\right\}
$$


endowed with the Fréchet-space topology inherited from the countable family of semi-norms $\|\cdot\|_{H^{1,2}\left(\Omega_{k} ; F\right)}, k \in \mathbb{N}$. Note that the set of smooth functions satisfying (2.1) lies dense in $\mathscr{H}$. Let $\left(\varphi_{l}\right)$ be a minimizing sequence for $E$ in $\mathscr{H}$, that is $E\left(\varphi_{l}\right) \rightarrow \inf _{\varphi \in \mathscr{H}} E(\varphi)$ as $l \rightarrow \infty$. We may assume that $E\left(\varphi_{l}\right) \leqq E\left(\varphi_{o}\right)<\infty$, uniformly in $l \in \mathbb{N}$.

By $\left(1^{\circ}\right)$ and since the energy density $e(\varphi)=|\nabla \varphi|^{2}+V(\varphi) \geqq 0$, for any set $\Omega_{k}$ we have

$$
\int_{\Omega_{k}}\left|\nabla \varphi_{l}\right|^{2} d x+C^{-1} \int_{\Omega_{k}}\left|\varphi_{l}\right|^{2} d x \leqq E\left(\varphi_{l} ; \Omega_{k}\right)+C \operatorname{vol}\left(\Omega_{k}\right) \leqq E\left(\varphi_{l}\right)+C \operatorname{vol}\left(\Omega_{k}\right)<\infty,
$$

uniformly in $l \in \mathbb{N}$. That is, $\left(\varphi_{l}\right)$ is bounded in $H^{1,2}\left(\Omega_{k} ; F\right)$ for any $k$. Extracting a diagonal subsequence, if necessary, we may assume that $\varphi_{l} \rightarrow \varphi$ weakly in $H^{1,2}\left(\Omega_{k} ; F\right)$ on any $\Omega_{k}$ and pointwise almost everywhere on $M$. Letting $\pi_{1}(M)$ act, since $\pi_{1}(M)$ is countable, we obtain the same conclusion for $\tilde{M}$. Thus, in particular, for any $[\omega] \in \pi_{1}(M)$ relation (2.1) is satisfied almost everywhere. Moreover, since any compact $\Omega \Subset \tilde{M}$ is covered by finitely many images of compact regions $\Omega_{o} \Subset M$ under covering transformations $\left[\omega_{1}\right], \ldots,\left[\omega_{m}\right] \in \pi_{1}(M)$, the weak limit $\varphi \in H_{\text {loc }}^{1,2}(\tilde{M} ; F)$; that is, $\varphi \in \mathscr{H}$. Finally, by weak lower semi-continuity of the $L^{2}$-norm, we have

$$
\int_{\Omega_{k}}|\nabla \varphi|^{2} d x \leqq \liminf _{l \rightarrow \infty} \int_{\Omega_{k}}\left|\nabla \varphi_{l}\right|^{2} d x
$$

while by Fatou's lemma, since $V$ is non-negative, we obtain

$$
\int_{\Omega_{k}} V(\varphi) d x \leqq \liminf _{l \rightarrow \infty} \int_{\Omega_{k}} V\left(\varphi_{l}\right) d x
$$

for all $k$. That is,

$$
E\left(\varphi ; \Omega_{k}\right) \leqq \liminf _{l \rightarrow \infty} E\left(\varphi_{l} ; \Omega_{k}\right) \leqq \liminf _{l \rightarrow \infty} E\left(\varphi_{l}\right)=\inf _{\varphi \in \mathscr{H}} E(\varphi),
$$

for any $k$. Upon letting $k \rightarrow \infty$ we see that $\varphi$ minimizes $E$ in $\mathscr{H}$, as desired.

The minimizer $\varphi$ may be referred to as a (generalized) ground state of the theory described by the energy $E$.

The proof of Theorem 2.1 may be extended to more general energy functionals defined in terms of integrals of energy densities

$$
e(\varphi)=F(\cdot, \varphi, \nabla \varphi),
$$

where $F \geqq 0$ is measurable in $x$, continuous in $\varphi$, convex in $\nabla \varphi$, coercive in the sense that

$$
F \geqq C^{-1}\left(|\nabla \varphi|^{p}+|\varphi|^{q}\right)-C
$$

with $p>1, q \geqq 1$, and is $\pi_{1}(M)$ - as well as $G$-invariant in the sense that

$$
F([\omega] x, g \varphi, g \nabla \varphi)=F(x, \varphi, \nabla \varphi)
$$

for all $[\omega] \in \pi_{1}(M), g \in G, \varphi \in \mathscr{H}$, and almost every $x \in \tilde{M}$; see Giaquinta [19], Morrey [20]. 


\section{Regularity Results for the Landau-Ginzburg Model}

As a special case of the preceding discussion, consider the $\mathbb{Z}_{2}$-bundle over $M_{\mathscr{L}}$ of Example 3. We look for functions $\varphi \in H_{\mathrm{loc}}^{1,2}\left(\tilde{M}_{\mathscr{L}}\right)$ that minimize the energy (1.43) subject to the holonomy constraint

$$
\varphi([\omega] x)=(-1)^{n(\omega, \mathscr{L})} \varphi(x)
$$

almost everywhere, for all $[\omega] \in \pi_{1}\left(M_{\mathscr{L}}\right)$. As indicated in Sect. 1 we would also like to study the effect of scaling with a factor $\varepsilon$. To do this, it will be convenient to look at

$$
E_{\varepsilon}(\varphi)=\frac{1}{2} \int_{M_{\mathscr{L}}}\left(\varepsilon|\nabla \varphi|^{2}+\frac{1}{\varepsilon} V(\varphi)\right) d x
$$

instead of (1.43).

Moreover, we look at general potentials $V$ sharing the qualitative properties of $V(\varphi)=\frac{1}{2}\left(|\varphi|^{2}-1\right)^{2}$; that is, we make the following assumptions:

(V1) $V: \mathbb{R} \rightarrow \mathbb{R}$ is of class $C^{\infty}$, non-negative and even, that is, $V(\varphi)=V(-\varphi)$;

(V2) $V(\varphi)=0$ if and only if $|\varphi|=1$;

(V3) $V^{\prime \prime}(\varphi)>0$, for $|\varphi| \geqq 1$;

(V4) $V^{\prime}(\varphi)-V^{\prime \prime}(\varphi) \varphi<0$, for $\varphi>0$.

Given $\mathscr{L}$, we may choose a region $\Omega \subset \mathbb{R}^{3}$ containing $\mathscr{L}$ and smooth surfaces $\mathscr{C}$ spanning $\mathscr{L}$, contained in $\Omega$. Let $\varphi_{o}$ be a harmonic function in $\Omega \backslash \mathscr{C}$ satisfying the boundary conditions

$$
\varphi_{o}=1 \text { on } \partial \Omega, \quad \varphi_{o}=0 \text { on } \mathscr{C},
$$

and let $\varphi_{o} \equiv 1$ outside $\Omega$. Extend $\varphi_{o}$ to the universal cover $\tilde{M}_{\mathscr{L}}$ of $M_{\mathscr{L}}$ by using (3.1). Then $\varphi_{0} \in H_{\text {loc }}^{1,2}\left(\widetilde{M}_{\mathscr{L}}\right)$; moreover, for any $\varepsilon>0$ we have

$$
E_{\varepsilon}\left(\varphi_{o}\right)<\infty \text {. }
$$

Finally, $\varphi_{o}$ satisfies (3.1) by construction. By Theorem 2.1, therefore, $E_{\varepsilon}$ admits a minimizer $\varphi_{\varepsilon} \in H_{\text {loc }}^{1,2}\left(\tilde{M}_{\mathscr{L}}\right)$ satisfying (3.1), for any $\varepsilon>0$. Our goal in this section will be to establish the following regularity properties for $\varphi_{\varepsilon}$. The proof invokes only standard tools in elliptic regularity theory.

Theorem 3.1. Suppose $V$ satisfies (V1)-(V3). Let $\varepsilon>0$. Then any minimizer $\varphi_{\varepsilon} \in H_{\mathrm{loc}}^{1,2}\left(\tilde{M}_{\mathscr{L}}\right)$ of $E_{\varepsilon}$, satisfying (3.1), has the properties:

1. $-1<\varphi_{\varepsilon}<1$ in $\tilde{M}_{\mathscr{L}}$.

2. $\varphi_{\varepsilon} \in C^{\infty}\left(\tilde{M}_{\mathscr{L}}\right)$, and for any $\delta>0$ and any multi-index $k$, there exists a constant $C=C(\delta, k)$ such that

$$
\left|\nabla^{k} \varphi_{\varepsilon}(x)\right| \leqq C \varepsilon^{-|k|},
$$

if

$$
\operatorname{dist}(x, \mathscr{L})=\inf \{|x-y| ; y \in \mathscr{L}\} \geqq \varepsilon \delta .
$$

3. Finally, $\left|\varphi_{\varepsilon}(x)\right| \rightarrow 1$ exponentially as $|x| \rightarrow \infty$ in the sense that, for any multi-index $k$, with constants $C=C(k, \varepsilon), c>0$, there holds

as $|x| \rightarrow \infty$.

$$
\left|\nabla^{k}\left(\left|\varphi_{\varepsilon}\right|-1\right)(x)\right| \leqq C(k, \varepsilon) \exp \left(\frac{-|x|}{c \varepsilon}\right)
$$


Proof. $\left(1^{\circ}\right)$ Given a minimizer $\varphi_{\varepsilon}$ of $E_{\varepsilon}$ we construct a comparison function $\bar{\varphi}_{\varepsilon}$ by letting

$$
\bar{\varphi}_{\varepsilon}(x)=\left\{\begin{array}{lll}
1, & \text { if } \quad \varphi_{\varepsilon} \geqq 1 \\
\varphi_{\varepsilon}(x), & \text { if } \quad-1 \leqq \varphi_{\varepsilon} \leqq 1 \\
-1, & \text { if } \quad \varphi_{\varepsilon} \leqq-1
\end{array}\right.
$$

Note that $\bar{\varphi}_{\varepsilon} \in H_{\text {loc }}^{1,2}\left(\tilde{M}_{\mathscr{L}}\right)$ and satisfies (3.1). Moreover, $\nabla \bar{\varphi}_{\varepsilon}(x)=0$ almost everywhere on the sets $\left\{x ; \bar{\varphi}_{\varepsilon}(x)= \pm 1\right\}$. In particular, $\nabla \bar{\varphi}_{\varepsilon}=0$ on the set where $\varphi_{\varepsilon} \neq \bar{\varphi}_{\varepsilon}$, and it follows that

$$
\begin{aligned}
0 \geqq E_{\varepsilon}\left(\varphi_{\varepsilon}\right)-E_{\varepsilon}\left(\bar{\varphi}_{\varepsilon}\right) & =\int_{\left\{x ; \varphi_{\varepsilon}(x) \neq \bar{\varphi}_{\varepsilon}(x)\right\}} \frac{\varepsilon}{2}\left|\nabla \varphi_{\varepsilon}\right|^{2} d x+\frac{1}{2 \varepsilon_{M_{\mathscr{L}}}} \int\left(V\left(\varphi_{\varepsilon}\right)-V\left(\bar{\varphi}_{\varepsilon}\right)\right) d x \\
& \geqq \frac{1}{2 \varepsilon_{M_{\mathscr{L}}}}\left(V\left(\varphi_{\varepsilon}\right)-V\left(\bar{\varphi}_{\varepsilon}\right)\right) d x \\
& =\frac{1}{2 \varepsilon} \int_{\left\{x ;\left|\varphi_{\varepsilon}(x)\right|>1\right\}} V\left(\varphi_{\varepsilon}\right) d x .
\end{aligned}
$$

But $V(\varphi)$ is positive for $|\varphi|>1$ and hence $\left|\varphi_{\varepsilon}\right| \leqq 1$ almost everywhere.

Now choose a smooth function $\varphi \in C_{o}^{\infty}\left(M_{\mathscr{L}}\right)$ whose support is contained in a simply connected compact region of $M_{\mathscr{L}}$ and extend $\varphi$ to $\tilde{M}_{\mathscr{L}}$ by (3.1). Then for $\delta \in \mathbb{R}$ the map $\varphi_{\varepsilon}+\delta \varphi$ is an admissible comparison function. Moreover, since $\varphi_{\varepsilon}$ is bounded, $V \in C^{\infty}$, and since $\varphi$ has compact support, the map $\delta \mapsto E_{\varepsilon}\left(\varphi_{\varepsilon}+\delta \varphi\right)$ is of class $C^{\infty}$. By minimality of $\varphi_{\varepsilon}$, then

$$
\frac{d}{d \delta} E_{\varepsilon}\left(\varphi_{\varepsilon}+\delta \varphi\right)_{\mid \delta=0}=\varepsilon \int_{M_{\mathscr{L}}}\left\langle\nabla \varphi_{\varepsilon}, \nabla \varphi\right\rangle d x+\frac{1}{2 \varepsilon} \int_{M_{\mathscr{L}}} V^{\prime}\left(\varphi_{\varepsilon}\right) \varphi d x=0 .
$$

Thus, upon integrating by parts in the first term and since $\varphi$ is essentially arbitrary, we infer that $\varphi_{\varepsilon}$ weakly solves the Euler-Lagrange equations

$$
-\varepsilon \Delta \varphi_{\varepsilon}+\frac{1}{2 \varepsilon} V^{\prime}\left(\varphi_{\varepsilon}\right)=0 \text { in } \tilde{M}_{\mathscr{L}}
$$

associated with $E_{\varepsilon}$.

Since $\left|\varphi_{\varepsilon}\right| \leqq 1$ uniformly, and since $V \in C^{\infty}$, it follows that $\left|V^{\prime}\left(\varphi_{\varepsilon}\right)\right| \leqq C$ uniformly with an absolute constant $C=\max _{|\varphi| \leqq 1}\left|V^{\prime}(\varphi)\right|$ independent of $\varepsilon$ and $\varphi_{\varepsilon}$.

Linear elliptic regularity theory then yields that $\varphi_{\varepsilon} \in H_{\text {loc }}^{2, p}\left(\tilde{M}_{\mathscr{L}}\right)$ for all $p<\infty$ (see for instance Gilbarg-Trudinger [21, Theorem 9.13]), whence by the Sobolev embedding theorem also $\varphi_{\varepsilon} \in C^{1, \alpha}\left(\tilde{M}_{\mathscr{L}}\right)$ for all $\alpha<1$. Thus $V^{\prime}\left(\varphi_{\varepsilon}\right) \in C^{1, \alpha}$, and hence $\varphi_{\varepsilon} \in C^{3, \alpha}$ for any $\alpha<1$. By iteration it follows that $\varphi_{\varepsilon} \in C^{\infty}$, as claimed. Finally, as a consequence of the strong maximum principle, $\varphi_{\varepsilon}$ cannot achieve the values +1 or -1 in $\tilde{M}_{\mathscr{L}}$. [Observe that $\varphi_{\varepsilon} \equiv 1$ or $\varphi_{\varepsilon} \equiv-1$ on $\tilde{M}_{\mathscr{L}}$ is impossible by the holonomy condition (3.1).]

$\left(2^{\circ}\right)$ Scale

$$
\hat{\varphi}_{\varepsilon}(x)=\varphi_{\varepsilon}(\varepsilon x) .
$$

Note that $\hat{\varphi}=\hat{\varphi}_{\varepsilon}$ satisfies the equation

$$
-\Delta \hat{\varphi}+\frac{1}{2} V^{\prime}(\hat{\varphi})=0
$$


in a domain $\tilde{M}_{\hat{\mathscr{L}}}$, the universal cover of $M_{\hat{\mathscr{L}}}=\mathbb{R}^{3} \backslash \hat{\mathscr{L}}$, where $\hat{\mathscr{L}}=\hat{\mathscr{L}}_{\varepsilon}=\mathscr{L} / \varepsilon$. Moreover, $|\hat{\varphi}| \leqq 1$ and hence from (3.3) and the local elliptic regularity theory (see for instance Gilbarg-Trudinger [21; Theorem 9.11]) we immediately obtain uniform local estimates $\left|\nabla^{k} \hat{\varphi}_{\varepsilon}(x)\right| \leqq C(k, \delta)$, if $\operatorname{dist}(x, \hat{\mathscr{L}}) \geqq \delta$, for any derivative of $\hat{\varphi}_{\varepsilon}$, that scale appropriately with $\varepsilon$.

\section{(3) Exponential Decay}

From $\left(2^{\circ}\right)$ it follows that - away from $\mathscr{L}-\left|\nabla \varphi_{\varepsilon}\right|$ is uniformly bounded for fixed $\varepsilon>0$.

Let $\Omega$ be a fixed region containing $\mathscr{L}$ as above. Then $\left|\nabla \varphi_{\varepsilon}\right| \leqq C_{o}$ uniformly outside $\Omega$; in particular, if $\left|\varphi_{\varepsilon}(x)\right| \leqq 1-2 \delta$ for some $x \notin \Omega, \delta>0$, then $\left|\varphi_{\varepsilon}(y)\right| \leqq 1-\delta$ for all $y \in B_{\delta C_{o}^{-1}}(x)$, and it follows that $V\left(\varphi_{\varepsilon}(y)\right) \geqq c>0$ for all such $y$ with a constant $c \sim \delta^{2}>0$. But $E_{\varepsilon}\left(\varphi_{\varepsilon}\right)<\infty$; thus $\left|\varphi_{\varepsilon}(x)\right| \rightarrow 1$ uniformly as $|x| \rightarrow \infty$.

By (V3) there exist constants $c_{0}>0, \delta_{0}>0$ such that $V^{\prime \prime}(\varphi) \geqq c_{o}>0$ for $1 \geqq|\varphi|$ $\geqq 1-\delta_{o}$. Choose $d_{o}$ such that $\left|\varphi_{\varepsilon}(x)\right| \geqq 1-\delta_{o}$ for $|x| \geqq d_{o}$. Differentiating (3.2) and multiplying by $\nabla \varphi_{\varepsilon}$, then we obtain that

$$
\begin{aligned}
0 & =-\Delta\left(\left|\nabla \varphi_{\varepsilon}\right|^{2}\right)+2\left|\nabla^{2} \varphi_{\varepsilon}\right|^{2}+\varepsilon^{-2} V^{\prime \prime}\left(\varphi_{\varepsilon}\right)\left|\nabla \varphi_{\varepsilon}\right|^{2} \\
& \geqq-\Delta\left(\left|\nabla \varphi_{\varepsilon}\right|^{2}\right)+\varepsilon^{-2} c_{o}\left|\nabla \varphi_{\varepsilon}\right|^{2} \text { for }|x| \geqq d_{o} .
\end{aligned}
$$

Since the fundamental solution to the operator $\left(-\Delta+c_{o} \varepsilon^{-2}\right)$ decays like $\exp \left(\frac{-\sqrt{c_{o}}|x|}{\varepsilon}\right)$ and since we already know that $\left|\nabla \varphi_{\varepsilon}\right|$ is uniformly bounded away from $\mathscr{L}$, this proves that $\left|\nabla \varphi_{\varepsilon}\right|$ decays exponentially at a rate proportional to $1 / \varepsilon$ as $|x| \rightarrow \infty$. Exponential decay of higher derivatives is obtained in a similar way.

Remark. The above argument shows that, in particular, for a system $\mathscr{L}$ of curves that can be decomposed into sub-systems $\mathscr{L}^{(1)}, \ldots, \mathscr{L}^{(n)}$ separated by a distance

$$
d_{\mathscr{L}}=\inf _{i \neq j} \operatorname{dist}\left(\mathscr{L}^{(i)}, \mathscr{L}^{(j)}\right) \gg 1
$$

the ground state energy for the system $\mathscr{L}$ decomposes into the ground state energies for the components $\mathscr{L}^{(j)}$ with error

$$
\left|\inf _{\varphi \in \mathscr{H} \mathscr{L}} E_{\mathscr{L}}(\varphi)-\sum_{j=1}^{n} \inf _{\varphi \in \mathscr{H} \mathscr{L}_{(j)}} E_{\mathscr{L}^{(j)}}(\varphi)\right|
$$

decaying exponentially at a rate proportional to $d_{\mathscr{L}} / \varepsilon$. Indeed, let $\varphi, \varphi^{(1)}, \ldots, \varphi^{(n)}$ denote, respectively, minimizers of $E_{\mathscr{L}}, E_{\mathscr{L}(1)}, \ldots, E_{\mathscr{L}(n)}$, and let $\tau^{(0)}, \tau^{(1)}, \ldots, \tau^{(n)}$ denote a smooth partition of unity such that $0 \leqq \tau^{(j)} \leqq 1, \tau^{(j)}(x)=1$ if $\operatorname{dist}\left(x, \mathscr{L}^{(j)}\right)$ $\leqq \frac{d_{\mathscr{L}}}{3}, \tau^{(j)}(x)=0$, if $\operatorname{dist}\left(x, \mathscr{L}^{(j)}\right) \geqq \frac{d_{\mathscr{L}}}{2}$. Then the functions $\psi^{(j)}=\left(1-\tau^{(j)}\right)+\tau^{(j)}|\varphi|$, extended equivariantly to $\tilde{M}_{\mathscr{L}(j)}$, are admissible comparison functions for $E_{\mathscr{L}(j)}$ and

$$
\sum_{j=1}^{n} E_{\mathscr{L}(j)}\left(\psi^{(j)}\right) \leqq E_{\mathscr{L}}(\varphi)+C \exp \left(-c d_{\mathscr{L}} / \varepsilon\right)
$$

by exponential decay of $\varphi$. 

Similarly, choosing $\psi=\tau^{(0)}+\sum_{j=1}^{n} \tau^{j}\left|\varphi^{(j)}\right|$ on $\mathbb{R}^{3} \backslash \mathscr{L}$, yields a comparison
function for $E_{\mathscr{L}}$ such that

$$
E_{\mathscr{L}}(\psi) \leqq \sum_{j=1}^{n} E_{\mathscr{L}(j)}\left(\varphi^{(j)}\right)+C \exp \left(-c d_{\mathscr{L}} / \varepsilon\right)
$$

Behaviour of $\varphi_{\varepsilon}$ near $\mathscr{L}$.

We summarize our results in the following

Theorem 3.2. Let $\varphi_{\varepsilon}$ be a minimizer of $E_{\varepsilon}$ in $H_{\text {loc }}^{1,2}\left(\tilde{M}_{\mathscr{L}}\right)$ satisfying (3.1) almost everywhere. Then

$$
\varphi_{\varepsilon}(x) \rightarrow 0 \quad \text { uniformly as } \operatorname{dist}(x, \mathscr{L}) \rightarrow 0 ;
$$

moreover, for any multi-indices $k$ and $l$ we have

$$
\left|\nabla_{\text {tan }}^{l} \nabla_{\text {nor }}^{k} \varphi_{\varepsilon}(x)\right| \leqq C \varepsilon^{-1 / 2-|l|} \operatorname{dist}(x, \mathscr{L})^{1 / 2-|k|},
$$

for derivatives in directions normal and tangent to $\mathscr{L}$, uniformly in $x \in \tilde{M}_{\mathscr{L}}$ such that $\operatorname{dist}(x, \mathscr{L}) \leqq \frac{\varepsilon}{2}$, with constants $C=C(k, l)$.

Proof. Given $x_{o} \in \hat{\mathscr{L}}=\mathscr{L} / \varepsilon$, let $\hat{\Omega}$ be a tubular neighbourhood of $\hat{\mathscr{L}}$ near $x_{o}$ and let $T: \hat{\Omega} \rightarrow C$ be parallel coordinates based on $\hat{\mathscr{L}}$ mapping $\hat{\Omega}$ diffeomorphically onto the cylinder $C=B \times]-1,1\left[\right.$, where $B=B_{1}\left(0 ; \mathbb{R}^{2}\right)$, such that $T\left(x_{o}\right)=0$ and $T(\hat{\Omega} \cap \hat{\mathscr{L}})$ $=\{0\} \times]-1,1\left[\right.$. Denote $S=T^{-1}$. Also let $\left(z_{o}, t\right)$ denote points in $\left.B \times\right]-1,1[$. Observe that $S, T \rightarrow$ id smoothly, if $\varepsilon \rightarrow 0$. Hence all estimates depending on bounds for derivatives of $S$ or $T$ will be uniform in $\varepsilon \leqq 1$. Identify $\mathbb{R}^{2} \cong \mathbb{C}$ and let $(z, t)$ $=\left(z_{1}+i z_{2}, t\right)$ denote points in $C$. We then obtain a coordinate chart for the double cover $\widetilde{\Omega}$ of $\widehat{\Omega}$ by letting $\dot{C}=(B \backslash\{0\}) \times]-1,1\left[\right.$ and $P: \dot{C} \ni(z, t) \mapsto S\left(z^{2}, t\right) \in \tilde{M}_{\mathscr{L}}$, with local inverse $Q=P^{-1}$. Let

$$
\tilde{\varphi}=\tilde{\varphi}_{\varepsilon}=\hat{\varphi}_{\varepsilon} \circ P
$$

be the representation of $\hat{\varphi}_{\varepsilon}(x)=\varphi_{\varepsilon}(\varepsilon x)$ in this standard coordinate chart. Observe that the holonomy condition (3.1) translates into the condition

for $\tilde{\varphi}$.

$$
\tilde{\varphi}(-z, t)=-\tilde{\varphi}(z, t)
$$

Note that, for any variation vector $\tilde{\psi}$ with compact support in $\dot{C}$ and satisfying (3.4), and setting $\hat{\psi}=\tilde{\psi} \circ Q, \hat{\psi}$ is an admissible variation of $\hat{\varphi}$ with support in $\hat{\Omega}=P(\dot{C})$. Hence, after substitution of variables, from (3.3) we obtain

$$
\begin{aligned}
0 & =\int_{\delta}\left[\langle\nabla \hat{\varphi}, \nabla \hat{\psi}\rangle+\frac{1}{2} V^{\prime}(\hat{\varphi}) \hat{\psi}\right] d x \\
& =\int_{\tilde{C}}\left\{[\langle\nabla(\tilde{\varphi} \circ Q), \nabla(\tilde{\psi} \circ Q)\rangle \circ P]+\frac{1}{2} V^{\prime}(\tilde{\varphi}) \tilde{\psi}\right\}|\operatorname{det}(D P)| d z d t .
\end{aligned}
$$

By the chain rule

$$
\begin{aligned}
\langle\nabla(\tilde{\varphi} \circ Q), \nabla(\tilde{\psi} \circ Q)\rangle \circ P & =\operatorname{trace}\left(D \tilde{\varphi}(D Q \circ P)(D \tilde{\psi}(D Q \circ P))^{t}\right) \\
& =\operatorname{trace}\left(D \tilde{\varphi}\left[(D P)^{t} D P\right]^{-1} D \tilde{\psi}^{t}\right) \\
& =\langle\nabla \tilde{\varphi}, A \nabla \tilde{\psi}\rangle
\end{aligned}
$$

with a smooth, symmetric coefficient matrix $A=\left[(D P)^{t} D P\right]^{-1}$. 
Note that in our coordinates

$$
A=\left(\begin{array}{ccc}
\frac{1}{4|z|^{2}} & 0 & 0 \\
0 & \frac{1}{4|z|^{2}} & 0 \\
0 & 0 & a^{2}
\end{array}\right)
$$

with a smooth function $a(z, t)$, tending to 1 uniformly as $\varepsilon \rightarrow 0$. Moreover,

$$
|\operatorname{det}(D P)|=4|z|^{2} a^{-1}(z, t) \text {. }
$$

Hence from (3.5) we obtain that, with $\nabla_{z}=\left(\frac{\partial}{\partial z_{1}}, \frac{\partial}{\partial z_{2}}\right)$, etc.,

$$
\int_{C}\left\{\nabla_{z} \tilde{\varphi} \nabla_{z} \tilde{\psi}+|z|^{2} b\left(z^{2}, t\right) \partial_{t} \tilde{\varphi} \partial_{t} \tilde{\psi}+|z|^{2} c\left(z^{2}, t\right) V^{\prime}(\tilde{\varphi}) \tilde{\varphi}\right\} d z d t=0
$$

for all $\tilde{\psi} \in C_{o}^{\infty}(\dot{C})$ satisfying (3.4), with smooth coefficients $b=4 a, c=4 a^{-1}>0$. Note that $V^{\prime}$ is odd, whence by (3.4) the integrand is invariant under rotation $z \mapsto-z$. Choose an arbitrary function $\tilde{\psi}$ with compact support in a half-cylinder and extend $\tilde{\psi}(-z, t)=-\tilde{\psi}(z, t)$ to the remaining half.

Then we obtain relation (3.6) for all such $\tilde{\psi}$. Thus $\tilde{\varphi}$ weakly solves the equation

$$
-\Delta_{z} \tilde{\varphi}-\partial_{t}\left(|z|^{2} b \partial_{t} \tilde{\varphi}\right)+|z|^{2} c V^{\prime}(\tilde{\varphi})=0 \text { in } \dot{C} .
$$

Observe that, although $b>0$, the presence of the factor $|z|^{2}$ in the second term makes this equation degenerate in $t$-direction. However, as we shall see in Lemma 3.3 below, we nevertheless obtain uniform local bounds for all derivatives of $\tilde{\varphi}=\hat{\varphi}_{\varepsilon} \circ P$ in $\dot{C}$. Hence the assertion of Theorem 3.2 follows from the chain rule.

Lemma 3.3. Suppose $\tilde{\varphi} \in L^{2}(\dot{C})$ with $|\tilde{\varphi}| \leqq 1$ and

$$
\int_{C}\left(\left|\nabla_{z} \tilde{\varphi}\right|^{2}+|z|^{2}\left|\nabla_{t} \tilde{\varphi}\right|^{2}\right) d z d t<\infty
$$

weakly solves (3.7) with holonomy constraint (3.4). Then $\tilde{\varphi} \in C^{\infty}(C), \tilde{\varphi}(z, t) \rightarrow 0$ as $|z| \rightarrow 0$, uniformly in $t$, and, for any multi-index $k$, and any $l \in \mathbb{N}$ we have

$$
\left|\nabla_{z}^{k} \tilde{\varphi}(z, t)\right| \leqq C(k), \quad\left|\nabla_{t}^{l} \tilde{\varphi}(z, t)\right| \leqq C(l)|z|,
$$

uniformly in $|z| \leqq \frac{1}{2},|t| \leqq \frac{1}{2}$.

Proof. First observe that $\tilde{\varphi}$ weakly solves (3.7) on the solid cylinder $C$. Indeed, let $\tilde{\psi} \in C_{o}^{\infty}(C)$ be an arbitrary testing function. Since $0 \in B$ has capacity 0 we may choose a sequence $\tau_{k}=\tau_{k}(z) \in C_{o}^{\infty}(B)$ of cut-off functions $0 \leqq \tau_{k} \leqq 1$ vanishing near 0 and such that $\tau_{k} \rightarrow 1$ in $H^{1,2}$ as $k \rightarrow \infty$ on the support of $\tilde{\psi}$. Let $\int \ldots=\int_{C} \ldots$ Multiplying (3.7) with $\tilde{\psi} \tau_{k}$ and integrating by parts we obtain

$$
\int\left\{\left[\nabla_{z} \tilde{\varphi} \nabla_{z} \tilde{\psi}+|z|^{2} b \partial_{t} \tilde{\varphi} \partial_{t} \tilde{\psi}+|z|^{2} c V^{\prime}(\tilde{\varphi}) \tilde{\psi}\right] \tau_{k}+\nabla_{z} \tilde{\varphi} \nabla_{z} \tau_{k} \tilde{\varphi}\right\} d z d t=0 .
$$

But since $\nabla_{z} \tilde{\varphi} \in L^{2}, \tilde{\psi} \in L^{\infty}$, while $\nabla_{z} \tau_{k} \rightarrow 0$ in $L^{2}$ as $k \rightarrow \infty$, the last integral vanishes as we let $k \rightarrow \infty$. On the other hand, upon letting $k \rightarrow \infty$ in the first integral, we arrive at 


$$
\int\left[\nabla_{z} \tilde{\varphi} \nabla_{z} \tilde{\psi}+|z|^{2} b \partial_{t} \tilde{\varphi} \partial_{t} \tilde{\psi}+|z|^{2} c V^{\prime}(\tilde{\varphi}) \tilde{\psi}\right] d z d t=0
$$

for all $\tilde{\psi} \in C_{o}^{\infty}(C)$. That is, $\tilde{\varphi}$ weakly solves (3.7) in $C$.

We next derive integral a-priori bounds for $\nabla_{z} \tilde{\varphi}$ and its derivatives. These can be obtained iteratively by testing (3.7) with $\nabla^{2 m} \tilde{\varphi} \tau^{2}, m=1,2, \ldots$, where $\tau \in C_{0}^{\infty}(C)$ is a smooth cut-off function, and integrating by parts. In order to bring out the essential ideas we simplify the computations by suppressing all terms involving derivatives of the cut-off function $\tau$ or the coefficients of the equation (which in any event only contribute lower order terms that can be estimated nicely at each step of the iteration in terms of quantities estimated in a previous step).

Moreover, to simplify the writing even more we only consider the case $b=c \equiv 1$. Thus, our equation reads

$$
-\Delta_{z} \tilde{\varphi}-|z|^{2} \Delta_{t} \tilde{\varphi}+|z|^{2} V^{\prime}(\tilde{\varphi})=0 .
$$

Suppose the induction hypothesis $\left|\nabla^{k}\left(\nabla_{z} \tilde{\varphi}\right)\right|+|z|\left|\nabla^{k}\left(\nabla_{t} \tilde{\varphi}\right)\right| \in L_{\text {loc }}^{2}(C)$ holds true for all $|k| \leqq m-1$. By our initial assumption about $\tilde{\varphi}$ this condition is in particular satisfied for $m=1$. Moreover, multiplying (3.7) by $\tilde{\varphi} \tau^{2}$ and using the fact that $|\tilde{\varphi}| \leqq 1$, we obtain the a-priori bound

$$
\begin{aligned}
& \int\left(\left|\nabla_{z} \tilde{\varphi}\right|^{2} \tau^{2}+|z|^{2}\left|\nabla_{t} \tilde{\varphi}\right|^{2} \tau^{2}\right) d z d t \\
& \quad \leqq \int\left(|z|^{2}\left|V^{\prime}(\tilde{\varphi}) \tilde{\varphi}\right| \tau^{2}+\left|\nabla_{z} \tilde{\varphi}\right| \tau|\tilde{\varphi}|\left|\nabla_{z} \tau\right|+|z|^{2}\left|\nabla_{t} \tilde{\varphi}\right| \tau|\tilde{\varphi}|\left|\nabla_{t} \tau\right|\right) d z d t \\
& \quad \leqq \frac{1}{2} \int\left(\left|\nabla_{z} \tilde{\varphi}\right|^{2} \tau^{2}+|z|^{2}\left|\nabla_{t} \tilde{\varphi}\right|^{2} \tau^{2}\right) d z d t+C \int\left(1+|\nabla \tau|^{2}\right) d z d t,
\end{aligned}
$$

for any $\tau \in C_{0}^{\infty}(C), \tau \geqq 0$.

Suppose $\left|\nabla^{m}\left(\nabla_{z} \tilde{\varphi}\right)\right|+|z|\left|\nabla^{m}\left(\nabla_{t} \tilde{\varphi}\right)\right| \in L^{2}$. Then upon testing (3.8) with $\nabla^{2 m} \tilde{\varphi} \tau^{2}$ and integrating by parts we obtain that

$$
\begin{aligned}
& \int\left\{\left|\nabla^{m}\left(\nabla_{z} \tilde{\varphi}\right)\right|^{2}+|z|^{2}\left|\nabla^{m}\left(\nabla_{t} \tilde{\varphi}\right)\right|^{2}\right\} \tau^{2} d z d t \\
& \quad \leqq C \int\left\{|z|\left|\nabla^{m-1}\left(\nabla_{t} \tilde{\varphi}\right) \nabla^{m}\left(\nabla_{t} \tilde{\varphi}\right)\right|+\left|\nabla^{m-1}\left(\nabla_{t} \tilde{\varphi}\right)\right|^{2}+\left|\nabla^{m}\left(|z|^{2} V^{\prime}(\tilde{\varphi})\right) \nabla^{m} \tilde{\varphi}\right|\right\} \tau^{2} d z d t+\ldots
\end{aligned}
$$

Note that the first and second terms on the right only arise if we evaluate contributions to $\left|\nabla^{m}\left(\nabla_{z} \tilde{\varphi}\right)\right|^{2}$ coming from operators $\nabla_{z}^{k} \nabla_{t}^{m-k}(k>0)$; that is, involving also $z$-derivatives. Hence after a change of order of differentiation these terms can be bounded

$$
\begin{aligned}
& \leqq C \int\left\{|z|\left|\nabla^{m-1}\left(\nabla_{z} \tilde{\varphi}\right)\right|\left|\nabla^{m}\left(\nabla_{t} \tilde{\varphi}\right)\right|+\left|\nabla^{m-1}\left(\nabla_{z} \tilde{\varphi}\right)\right|^{2}\right\} \tau^{2} d z d t \\
& \leqq \frac{1}{2} \int|z|^{2}\left|\nabla^{m}\left(\nabla_{t} \tilde{\varphi}\right)\right|^{2} \tau^{2} d z d t+C \int\left|\nabla^{m-1}\left(\nabla_{z} \tilde{\varphi}\right)\right|^{2} \tau^{2} d z d t
\end{aligned}
$$

Here, we have also used the binomial inequality $2|a b|=2\left|\varepsilon a\left(\frac{b}{\varepsilon}\right)\right| \leqq \varepsilon^{2} a^{2}+\frac{b^{2}}{\varepsilon^{2}}$, for any $\varepsilon>0, a, b \in \mathbb{R}$. Now the first of these terms can be absorbed on the left of (3.9) while the second term of (3.10) and the last term in (3.9) are bounded by induction assumption.

To remove the assumption $\left|\nabla^{m}\left(\nabla_{z} \tilde{\varphi}\right)\right| \in L^{2}$ we must replace differentials by difference quotients. See for instance Giaquinta [19]. Hence $\left|\nabla^{m}\left(\nabla_{z} \tilde{\varphi}\right)\right| \in L_{1 \mathrm{loc}}^{2}(C)$ for all $m \in \mathbb{N}$, with a-priori bounds on any region $\Omega \Subset C$ depending only on $m$ and $\Omega$. Therefore, by the Sobolev embedding theorem, $\nabla_{z} \tilde{\varphi} \in C^{\infty}$, with uniform local bounds

$$
\left|\nabla^{k}\left(\nabla_{z} \tilde{\varphi}\right)\right| \leqq C(k)
$$


for any multi-index $k$ and $|z|,|t| \leqq \frac{1}{2}$. But now, in particular, (3.4) implies that $\tilde{\varphi}(0, t)=0$ for all $|t|<1$ and, moreover, that

$$
\tilde{\varphi}(z, t) \rightarrow 0 \quad \text { as } \quad z \rightarrow 0 \text {, uniformly in } t .
$$

Thus, $\nabla_{t}^{l} \varphi(0, t)=0$ for all $|t|<1$ and all $l \in \mathbb{N}$, and by the mean value theorem

$$
\left|\nabla_{t}^{l} \tilde{\varphi}(z, t)\right| \leqq|z| \sup \left|\nabla_{t}^{l}\left(\nabla_{z} \tilde{\varphi}\right)\right| \leqq C|z|
$$

for all $|z| \leqq \frac{1}{2}$, with $C=C(l)$.

\section{Connections with Minimal Surface Theory}

For small $\varepsilon>0$, the functional $E_{\varepsilon}$ may be regarded as a singular perturbation of the "pure" potential term $\frac{1}{2 \varepsilon} \int V(\varphi) d x$ with the preferred rest states $\varphi=+1, \varphi=-1$. The additional term $\frac{\varepsilon}{2} \int|\nabla \varphi|^{2} d x$ is related to the "smoothness" and "size" of the "interface" between regions where $\varphi \approx \pm 1$. In particular, as $\varepsilon \rightarrow 0$, minimizers of $E_{\varepsilon}$ will arrange themselves in a pattern where the area of this interface becomes minimal for the prescribed boundary condition or constraint. In this way, minimal or constant mean curvature hypersurfaces, arising e.g. as boundaries between different phases of two-phase fluids, can be understood; see for instance Modica [22]. His work draws from earlier ideas of Modica-Mortola [23] and of De Giorgi on " $\Gamma$-convergence" and minimal surfaces, and from work by Gurtin [24] on the "gradient theory" of phase transitions. Related results were obtained by KohnSternberg [25]. See also Anzellotti-Baldo-Visintin [26], Baldo [27], LuckhausModica [28], and the references in the above papers. For our problem, we observe a similar relation between minimizers of $E_{\varepsilon}$ for small $\varepsilon>0$ and minimal surfaces spanning $\Gamma$. In fact, for knotted curves $\mathscr{L}$, our results seem to imply a new proof for the existence of embedded minimal surface spanning $\mathscr{L}$, first established by Hardt-Simon [34]. Consider minimizers $\varphi_{\varepsilon}$ of $E_{\varepsilon}$, satisfying the holonomy constraint (3.1). We would like to study the behaviour of $\varphi_{\varepsilon}$ as $\varepsilon \rightarrow 0$.

For $\Omega \subset \mathbb{R}^{n}$ denote $B V(\Omega)$ the space of $L^{1}$-functions $u: \Omega \rightarrow \mathbb{R}$ with bounded variation

$$
\int_{\Omega}|D u|=\sup \left\{\int_{\Omega} \sum_{i=1}^{n} u \partial_{i} g^{i} d x ; g=\left(g^{1}, \ldots, g^{n}\right) \in C_{o}^{\infty}\left(\Omega ; \mathbb{R}^{n}\right),|g| \leqq 1\right\} ;
$$

see e.g. Giusti [29]. Following Modica [22], we let

$$
W(\varphi)=\int_{0}^{\varphi} \sqrt{V(\varrho)} d \varrho
$$

and define a functional

$$
E_{o}(\varphi)=\int_{M_{\mathscr{L}}}|D(W(\varphi))| .
$$

Note that for smooth $\varphi$ the functionals $E_{o}$ and $E_{\varepsilon}$ are related by

$$
E_{o}(\varphi)=\int_{M_{\mathscr{L}}} \sqrt{V(\varphi)}|\nabla \varphi| d x \leqq \frac{1}{2} \int_{M_{\mathscr{L}}}\left[\varepsilon|\nabla \varphi|^{2}+\frac{1}{\varepsilon} V(\varphi)\right] d x=E_{\varepsilon}(\varphi),
$$


for any $\varepsilon>0$. In particular,

$$
\inf E_{o} \leqq E_{o}\left(\varphi_{\varepsilon}\right) \leqq E_{\varepsilon}\left(\varphi_{\varepsilon}\right)=\inf E_{\varepsilon}
$$

for any $\varepsilon>0$, where we take the infimum with respect to $\varphi \in H_{\text {loc }}^{1,2}\left(\tilde{M}_{\mathscr{L}}\right)$ satisfying the holonomy constraint. Since $\left|\varphi_{\varepsilon}\right| \leqq 1$ we have $\left|W\left(\varphi_{\varepsilon}\right)\right| \leqq C$; moreover, we will show that

$$
\int_{M_{\mathscr{L}}}\left|D W\left(\varphi_{\varepsilon}\right)\right|=E_{o}\left(\varphi_{\varepsilon}\right) \leqq E_{\varepsilon}\left(\varphi_{\varepsilon}\right) \leqq C<\infty,
$$

uniformly in $\varepsilon>0$; see Theorem 4.2 below. Hence the family $\left(W\left(\varphi_{\varepsilon}\right)\right)_{\varepsilon>0}$ is uniformly locally bounded in $B V$ and therefore relatively compact, locally, in $L^{1}$ (see Giusti [29, Theorem 1.19]). Since $W^{\prime} \geqq c>0$ in any interval $[-1+\delta, 1-\delta]$, $\delta>0$, it follows that also $\left(\varphi_{\varepsilon}\right)_{\varepsilon}>0$ is relatively compact in $L^{1}$, locally. Any $L^{1}$ limit point $\varphi_{0}$ will satisfy the relation $V\left(\varphi_{0}\right)=0$, that is, $\left|\varphi_{0}\right|=1$ almost everywhere. Moreover, the holonomy constraint (3.1) will be preserved. Hence, on $\tilde{M}_{\mathscr{L}}$, the function $\varphi_{0}$ must be discontinuous across some surface $F_{0}=\operatorname{supp}\left(D \varphi_{0}\right)$. By lower semi-continuity of $E_{0}$ with respect to local $L^{1}$-convergence (see Giusti [29]) we also have that

$$
\begin{aligned}
E_{0}\left(\varphi_{0}\right) & =\int_{\mathcal{M}_{\mathscr{L}}}\left|D W\left(\varphi_{0}\right)\right|=\left(\int_{0}^{1} \sqrt{V(\varrho)} d \varrho\right) \cdot \int_{M_{\mathscr{L}}}\left|D \varphi_{0}\right|=\left(\int_{-1}^{1} \sqrt{V(\varrho)} d \varrho\right) \cdot \operatorname{area}\left(F_{0}\right) \\
& \leqq \liminf _{\varepsilon \rightarrow 0} E_{0}\left(\varphi_{\varepsilon}\right) \leqq \liminf _{\varepsilon \rightarrow 0} E_{\varepsilon}\left(\varphi_{\varepsilon}\right),
\end{aligned}
$$

and hence by Theorem 4.2 below that $F_{0}$ is a minimal surface spanning $\mathscr{L}$, see for instance Giusti [29, p. 5f.]. (The above argument is modelled on a similar reasoning by Modica [22].)

The relations between minimizers of $E_{\varepsilon}$ and minimal surfaces, however, seem to go much deeper than what we observed above. Let us first recall how the differential properties of minimal surfaces, represented by minimizers $\varphi_{o}$ of $E_{o}$, are derived. Let $\tau: \tilde{M}_{\mathscr{L}} \rightarrow T \tilde{M}_{\mathscr{L}}$ be a $C^{2}$-vector field vanishing in a neighbourhood of $\mathscr{L}$, and invariant under covering transformations in the sense that

$$
\tau([\omega] x)=\tau(x), \forall[\omega] \in \pi_{1}\left(M_{\mathscr{L}}\right), x \in \tilde{M}_{\mathscr{L}},
$$

where we identify the tangent spaces $T_{x} \tilde{M}_{\mathscr{L}}$ and $T_{[\omega] x} \tilde{M}_{\mathscr{L}}$. Here it will suffice to study vector fields $\tau$ supported on a simply connected bounded region $\Omega \subset M_{\mathscr{L}}$ that we extend equivariantly to $\tilde{M}_{\mathscr{L}}$, using (4.5). In local coordinates we may regard $\tau$ as a map in $C^{2}\left(\Omega ; \mathbb{R}^{3}\right)$.

We use $\tau$ to define a variation of the independent variables $T_{\delta}=(\mathrm{id}+\delta \tau): \tilde{M}_{\mathscr{L}}$ $\rightarrow \tilde{M}_{\mathscr{L}}$, invariant under $\pi_{1}\left(M_{\mathscr{L}}\right)$. Note that for $|\delta| \ll 1$ the map $T_{\delta}$ is a diffeomorphism of $\tilde{M}_{\mathscr{L}}$ onto itself. Given a minimizer $\varphi_{o}$ of $E_{o}$, we compute

$$
\frac{d}{d \delta} E_{o}\left(\varphi_{o} \circ(\mathrm{id}+\delta \tau)^{-1}\right)_{\mid \delta=0}=0 .
$$

By the chain rule (see Giusti [29, Lemma 10.1] for a rigorous derivation)

$$
\begin{aligned}
E_{o}\left(\varphi_{o} \circ T_{\delta}^{-1}\right)=\int_{\tilde{M}_{\mathscr{L}}}\left|\left(\left(D W\left(\varphi_{o}\right)\right) \circ T_{\delta}^{-1}\right) \cdot\left(D T_{\delta}^{-1}\right)\right| \\
\quad=\int_{\dot{M}_{\mathscr{L}}}\left|D W\left(\varphi_{o}\right)\left[\left(D T_{\delta}^{-1}\right) \circ T_{\delta}\right] \| \operatorname{det}\left(D T_{\delta}\right)\right| .
\end{aligned}
$$


Now

$$
\left(D T_{\delta}^{-1}\right) \circ T_{\delta}=\left(D T_{\delta}\right)^{-1}=\mathrm{id}-\delta D \tau+0\left(\delta^{2}\right), \operatorname{det}\left(D T_{\delta}\right)=1+\delta \operatorname{div} \tau+0\left(\delta^{2}\right),
$$

so that (Giusti $[29 ;(10.5)]$ )

$$
E_{o}\left(\varphi_{o} \circ T_{\delta}^{-1}\right)=E_{o}\left(\varphi_{o}\right)+\delta \int_{M_{\mathscr{L}}}\left|D W\left(\varphi_{o}\right)\right|(\operatorname{div} \tau-\langle v, D \tau \nu\rangle)+0\left(\delta^{2}\right),
$$

where $v$ is a smooth extension of the normal " $v=\frac{\nabla W\left(\varphi_{o}\right)}{\left|\nabla W\left(\varphi_{o}\right)\right|}$, to the minimal
surface $\operatorname{supp}\left(D \varphi_{o}\right)$. It follows that

$$
\int_{M_{\mathscr{L}}}\left|D W\left(\varphi_{o}\right)\right|(\operatorname{div} \tau-\langle v, D \tau v\rangle)=0,
$$

for all $\tau \in C^{2}$ having compact support in a simply connected region $\Omega \subset M_{\mathscr{L}}$. Let $\psi \in C_{o}^{\infty}(\Omega)$. For $\tau=\nu \psi$ we then obtain

$$
\langle v, D \tau v\rangle=v \cdot \nabla \psi, \operatorname{div} \tau=\operatorname{trace}(D v) \psi+v \cdot \nabla \psi,
$$

and hence that

$$
\int_{M_{\mathscr{L}}} \operatorname{trace}(D v)\left|D W\left(\varphi_{o}\right)\right| \psi d x=0
$$

for all $\psi \in C_{o}^{\infty}(\Omega)$. Note that

$$
\operatorname{trace}(D v)=2 H
$$

is twice the mean curvature of the surface represented by $\varphi_{o}$. Since $\psi$ is arbitrary, from (4.6) we thus obtain the well-known fact that the mean curvature of a minimal surface vanishes.

The minimizers $\varphi_{\varepsilon}$ of $E_{\varepsilon}$ enjoy a similar property. First observe the following result, again due to Modica [30].

Theorem 4.1. Suppose $n<8, V \in C^{2}, V \geqq 0, V(\varphi)=0$ if and only if $|\varphi|=1$. Let $u \in C^{3}\left(\mathbb{R}^{n}\right)$ with $-1<u<1$ satisfy (3.2) - for $\varepsilon=1$ - and be locally minimal in the sense that

$$
E(u+\varphi) \geqq E(u) \text { for all } \varphi \in C_{o}^{\infty}\left(\mathbb{R}^{n}\right) .
$$

Then after a rotation of coordinates

$$
u\left(x_{1}, \ldots, x_{n}\right)=\bar{u}\left(x_{1}\right)
$$

for some solution $\bar{u}$ of (3.2).

Actually, in the one-dimensional case, upon testing Eq. (3.2)

with $\frac{d}{d x_{1}} \bar{u}$, we obtain that

$$
-\frac{d^{2}}{d x_{1}^{2}} \bar{u}+\frac{1}{2} V^{\prime}(\bar{u})=0
$$

$$
\left|\frac{d}{d x_{1}} \bar{u}\right|^{2}-V(\bar{u})=\text { const } .
$$

Moreover, if $\bar{u}$ is locally minimal, this constant must vanish. Hence, possibly after a reflection $x_{1} \mapsto-x_{1}$, we have

$$
\frac{d}{d x_{1}} \bar{u}=\bar{u}^{\prime}=\sqrt{V(\bar{u})}
$$


and

$$
\left|\bar{u}^{\prime}\right|^{2}=\sqrt{V(\bar{u})} u^{\prime}=(W(\bar{u}))^{\prime}
$$

Thus

$$
\int_{-\infty}^{\infty}\left|\bar{u}^{\prime}\right|^{2} d t=\int_{-\infty}^{\infty} V(\bar{u}) d t=W(1)-W(-1)=\int_{-1}^{1} \sqrt{V(\varrho)} d \varrho=: C_{0} .
$$

We let $u_{n}(x)=\varphi_{\varepsilon_{n}}\left(\varepsilon_{n} x+x_{n}\right)$ for sequences $\varepsilon_{n} \searrow 0,\left(x_{n}\right)$ converging to a point on $\operatorname{supp}\left(D \varphi_{o}\right) \backslash \mathscr{L}$ such that $\left|u_{n}(0)\right| \leqq C<1$. Then, by Theorem $3.1,\left(u_{n}\right)$ is equi-bounded and equi-continuous in $C^{1}$, hence converges in $C^{1}$ to a solution $u \in C^{\infty}\left(\mathbb{R}^{3}\right)$ of (3.2) for $\varepsilon=1$ with $+-1<u<1$. Moreover, since $\varphi_{\varepsilon_{n}}$ is minimal, so is $u_{n}$, for all $n$, and hence $u$. Theorem 4.1 now implies that $|\nabla u|=\sqrt{V(u)} \neq 0$ and hence that $\left|\nabla u_{n}(x)\right| \neq 0$ in $B_{R \varepsilon_{n}}\left(x_{n}\right)$ for any $R>0$, if $n$ is sufficiently large. Hence we may define a smooth vector field $v_{\varepsilon}=\frac{\nabla \varphi_{\varepsilon}}{\left|\nabla \varphi_{\varepsilon}\right|}$, normal to the "approximate minimal surfaces" $\varphi_{\varepsilon}=$ const. For $\tau=\tau_{\varepsilon}=\psi v_{\varepsilon}$, where $\psi \in C_{o}^{\infty}(\Omega)$ on a simply connected sub-domain $\Omega \subset M_{\mathscr{L}}$, again let $T_{\delta}=(\mathrm{id}+\delta \tau)$ and consider $E_{\varepsilon}\left(\varphi_{\varepsilon} \circ T_{\delta}^{-1}\right)$. By the chain rule, as above we obtain

$$
0=\frac{d}{d \delta} E_{\varepsilon}\left(\varphi_{\varepsilon} \circ T_{\delta}^{-1}\right)=\int_{M_{\mathscr{L}}}\left(-\varepsilon \operatorname{trace}\left(\left(D \varphi_{\varepsilon} D \tau\right)^{t} D \varphi_{\varepsilon}\right)+e_{\varepsilon}\left(\varphi_{\varepsilon}\right) \operatorname{div} \tau\right) d x
$$

where

$$
e_{\varepsilon}(\varphi)=\frac{\varepsilon}{2}|\nabla \varphi|^{2}+\frac{1}{2 \varepsilon} V(\varphi)
$$

With our choice of $\tau=\psi v_{\varepsilon}$ we may simplify this expression as follows. Note that

$$
\operatorname{div} \tau=\operatorname{trace}\left(D v_{\varepsilon}\right) \psi+v_{\varepsilon} \cdot \nabla \psi, \quad \operatorname{trace}\left(\left(D \varphi_{\varepsilon} D \tau\right)^{t} D \varphi_{\varepsilon}\right)=\left|\nabla \varphi_{\varepsilon}\right|^{2} v_{\varepsilon} \cdot \nabla \psi
$$

Hence if we write

$$
\operatorname{trace}\left(D v_{\varepsilon}\right)=2 H_{\varepsilon}
$$

for the mean curvature of the level surfaces $\varphi_{\varepsilon}=$ const, we obtain the equation

$$
\int_{M_{\mathscr{L}}} 2 H_{\varepsilon} e_{\varepsilon}\left(\varphi_{\varepsilon}\right) \psi d x=\int_{M_{\mathscr{L}}}\left(\frac{\varepsilon}{2}\left|\nabla \varphi_{\varepsilon}\right|^{2}-\frac{1}{2 \varepsilon} V\left(\varphi_{\varepsilon}\right)\right) v_{\varepsilon} \cdot \nabla \psi d x,
$$

analogous to (4.6).

Finally, we may choose a function $\psi$ on the surface $\varphi_{\varepsilon}=0$ and extend $\psi$ by solving the ordinary differential equation $v_{\varepsilon} \cdot \nabla \psi=0$; that is, extend $\psi=$ const along the trajectories $t \mapsto \Phi(x, t)$ of the flow given by $\frac{\partial}{\partial t} \Phi=v_{\varepsilon} \circ \Phi$. Then the righthand side in (4.7) vanishes, and we obtain that in the mean along the trajectories of $\Phi$ - with weight factor $e_{\varepsilon}\left(\varphi_{\varepsilon}\right)$ - the mean curvature of the surfaces $\varphi_{\varepsilon}=$ const vanishes. The same conclusion applies pointwise - on every leaf $\varphi_{\varepsilon}=$ const - where

$$
\varepsilon^{2}\left|\nabla \varphi_{\varepsilon}\right|^{2}-V\left(\varphi_{\varepsilon}\right)=0
$$

as in the one-dimensional case. 
We may also use the 1-dimensional solution $\bar{u}$ of (3.2) to construct comparison functions $\psi_{\varepsilon}$ for $E_{\varepsilon}$, yielding bounds for the ground state energy in terms of geometric data of the boundary $\mathscr{L}$. This will also complete the proof of (4.3).

Theorem 4.2. Let $F_{0}$ be a least area minimal surface spanning $\mathscr{L}, C_{0}$ $=\int_{-1}^{1} \sqrt{V(\varphi)} d \varphi$. There exists a constant $C>0$ such that

$$
\inf _{\varphi} E_{\varepsilon}(\varphi) \leqq C_{0} \operatorname{area}\left(F_{0}\right)+C \varepsilon \text { length }(\mathscr{L})+O\left(\varepsilon^{2}\right) .
$$

Theorem 4.2 is related to results by Almgren-Browder-Lieb [17] and BrézisCoron-Lieb [16].

Proof. Given $\varepsilon_{0}>\varepsilon>0$, for $x \in \mathbb{R}^{3} \backslash \mathscr{L}$ let

$$
\bar{\varphi}_{\varepsilon}(x)= \begin{cases}\bar{u}\left(\frac{\operatorname{dist}\left(x, F_{0}\right)}{\varepsilon}\right), & \text { if } \quad \operatorname{dist}\left(x, F_{0}\right) \leqq \varepsilon_{0} \\ \bar{u}\left(\frac{\varepsilon_{0} \operatorname{dist}\left(x, F_{0}\right)}{\varepsilon\left(2 \varepsilon_{0}-\operatorname{dist}\left(x, F_{0}\right)\right)}\right), & \text { if } \varepsilon_{0} \leqq \operatorname{dist}\left(x, F_{0}\right)<2 \varepsilon_{0} \\ 1, & \text { else, }\end{cases}
$$

and extend $\bar{\varphi}_{\varepsilon}$ equivariantly to $\tilde{M}_{\mathscr{L}}$. Then, since $\left|\nabla\left(\operatorname{dist}\left(x, F_{0}\right)\right)\right|=1$ almost everywhere, we have

$$
\inf E_{\varepsilon}(\varphi) \leqq E_{\varepsilon}\left(\bar{\varphi}_{\varepsilon}\right)=\frac{1}{2 \varepsilon} \underset{\left\{x ; \operatorname{dist}\left(x, F_{0}\right) \leqq \varepsilon_{0}\right\}}{\int}\left(\left|\bar{u}^{\prime}\right|^{2}+V(\bar{u})\right) d x+R(\varepsilon)
$$

with error $R(\varepsilon)$ decaying exponentially fast as $\varepsilon \rightarrow 0$. Recall that $\left|\bar{u}^{\prime}\right|^{2}=V(\bar{u})$. Let $v$ be a unit normal vector field on $F_{0}$. Decompose

$$
\Omega=\left\{x ; \operatorname{dist}\left(x ; F_{0}\right) \leqq \varepsilon_{0}\right\}=\Omega_{1} \cup \Omega_{2},
$$

where

$$
\Omega_{1}=\left\{x=z+t v(z) ; z \in F_{0},|t| \leqq \varepsilon_{0}\right\} .
$$

By a change of variables $\Phi(z, t)=z+t v(z)$,

$$
E_{\varepsilon}\left(\bar{\varphi}_{\varepsilon} ; \Omega_{1}\right)=\frac{1}{\varepsilon} \int_{\Omega_{1}} V\left(\bar{u}\left(\frac{\operatorname{dist}\left(x, F_{0}\right)}{\varepsilon}\right)\right) d x=\int_{-\frac{\varepsilon_{0}}{\varepsilon}}^{\frac{\varepsilon_{0}}{\varepsilon}} \int_{F_{0}} V(\bar{u}(t))|\operatorname{det}(D \Phi(z, \varepsilon t))| d z d t .
$$

Now

$$
|\operatorname{det}(D \Phi(z, s))|=1+s H(z)+s^{2} K(z)
$$

with $H$ and $K$ denoting the mean curvature and the Gauss curvature of $F_{0}$, respectively. Since $H=0, K \leqq 0$, it follows that

$$
E_{\varepsilon}\left(\bar{\varphi}_{\varepsilon} ; \Omega_{1}\right) \leqq C_{0} \operatorname{area}\left(F_{0}\right) \text {. }
$$

On $\Omega_{2}$ we may introduce coordinates based on an orthogonal frame $\left(e_{1}, e_{2}, e_{3}\right)$ moving along $\mathscr{L}$, with $e_{1}$ tangent to $\mathscr{L}$. Let $s$ denote arc-length on $\mathscr{L}$, 
parametrized by $\gamma$, and

$$
x=\gamma(s)+a e_{2}(s)+b e_{3}(s)=\Psi(s, a, b) .
$$

Then

$$
D \Psi(s, a, b)=\left(\begin{array}{ccc}
1+a e_{21}^{\prime}+b e_{31}^{\prime} & 0 & 0 \\
b e_{32}^{\prime} & 1 & 0 \\
a e_{23}^{\prime} & 0 & 1
\end{array}\right)
$$
where $e_{2}^{\prime}=\left(e_{21}^{\prime}, e_{22}^{\prime}, e_{23}^{\prime}\right)$ denote the components of $e_{2}^{\prime}=\frac{d}{d s} e_{2}$ in the frame
$\left(e_{1}, e_{2}, e_{3}\right)$, etc. That is,

$$
|\operatorname{det} D \Psi(s, a, b)|=\left|1+a e_{21}^{\prime}+b e_{31}^{\prime}\right| \leqq 1+\varrho \kappa,
$$

where

$$
\varrho=\sqrt{a^{2}+b^{2}}=\operatorname{dist}(\Psi(s, a, b), \mathscr{L})
$$

and

$$
\kappa=\sqrt{\left|e_{21}^{\prime}\right|^{2}+\left|e_{31}^{\prime}\right|^{2}}
$$

is the curvature of $\mathscr{L}$. Let $\bar{\kappa}=\sup _{s} \kappa(s)$. In these coordinates now

$$
\begin{aligned}
& \frac{1}{\varepsilon} \int_{\Omega_{2}} V\left(\bar{u}\left(\frac{\operatorname{dist}\left(x, F_{0}\right)}{\varepsilon}\right)\right) d x=\frac{\pi}{\varepsilon} \int_{0}^{\varepsilon_{0}} \int_{\mathscr{L}} V\left(\bar{u}\left(\frac{\varrho}{\varepsilon}\right)\right)|\operatorname{det}(D \Psi)| \varrho d \varrho d s \\
& \leqq \varepsilon \pi \int_{0}^{\varepsilon_{0} / \varepsilon} \int_{\mathscr{L}} V(\bar{u}(t))(1+\varepsilon t \kappa) t d t d s \leqq C_{1} \varepsilon \operatorname{length}(\mathscr{L})+C_{2} \varepsilon^{2} \bar{\kappa} \text { length }(\mathscr{L}) .
\end{aligned}
$$

The functions $\bar{\varphi}_{\varepsilon}$ constructed above, hopefully, give a good approximation to the actual minimizers $\varphi_{\varepsilon}$. A precise result in this regard, however, remains to be found.

\section{Uniqueness and Stability of Minimizers}

The results in this section seem to be related to results by Bronsard-Kohn [31], Carr-Pego [32] on the "dynamical" behavior of minimizers of our functional $E_{\varepsilon}$, however, under different boundary conditions. See also Alikakos-Bates-Fusco [33] for related results in the context of the Cahn-Hilliard equation.

Note that the construction in the proof of Theorem 4.2 shows that (even if we disregard the obvious symmetry $\varphi \rightarrow-\varphi$ ) minimizers to $E_{\varepsilon}$ need not be unique, in general. In fact, it may happen that $\mathscr{L}$ spans several geometrically distinct least area surfaces, possibly of different topological types. This is clear in the 2-dimensional case, where the notions "curve" and "surface" have to be appropriately replaced by the notions "point" and "curve:" Take a configuration consisting of the vertices of a square, for example. But non-uniqueness also occurs in higher dimensions. As an example, consider the curve $\mathscr{L}$ consisting of two plane, co-axial circles of the same radius. In this case, depending on the distance $d$ of the circles from another, besides the obvious locally area-minimizing surface that consists of a pair of discs spanning $\mathscr{L}$, there may also be a relatively minimal doubly connected surface, a catenoid, spanning $\mathscr{L}$. Moreover, for small $d$, the 
catenoid will be absolutely area minimizing among surfaces spanning $\mathscr{L}$, while for large $d$ the surface consisting of a pair of discs will be. Thus (everything depends smoothly on $d$ ) for a certain intermediate value of $d$ there will be two geometrically (even topologically) distinct least area surfaces spanning $\mathscr{L}$. Hence, also $E_{\varepsilon}$ may admit many relative minima for small $\varepsilon>0$.

It therefore seems reasonable to limit our investigation of uniqueness and stability questions to a class of boundary curves $\mathscr{L}$ that span a unique minimal surface. One particular such class is the class of plane curves. (In two dimensions, we consider singular points on a line; also in this case, the stability result below does not seem to be known.)

For such curves, lying in the plane $y=0$, say, one may expect minimizers $\varphi_{\varepsilon}$ of $E_{\varepsilon}$ to enjoy the reflexion symmetry $\varphi_{\varepsilon}(x,-y)=\varphi_{\varepsilon}(x, y)$, where we denote $x=\left(x_{1}, x_{2}\right)$, and $\bar{x}=(x, y)$ the coordinates in $\mathbb{R}^{3}$. (For simplicity we fix $\varepsilon=1$ and omit the index $\varepsilon$ from now on.)

Theorem 5.1. Suppose $\mathscr{L}$ lies in the plane $y=0$. Then there exists a minimizer $\varphi \in H_{\mathrm{loc}}^{1,2}\left(\tilde{M}_{\mathscr{L}}\right)$ of $E$ satisfying the holonomy constraint (3.1) and such that $\varphi(x, y)$ $=\varphi(x,-y), 0 \leqq \varphi<1$ on $M_{\mathscr{L}}$, and $\varphi$ is non-decreasing in $|y|$.

Proof. Let $\varphi$ be a minimizer of $E$ satisfying (3.1). We symmetrize $\varphi$ over the fundamental domain $M_{\mathscr{L}}$ by letting $\varphi^{*}$ be the unique function such that $\varphi^{*}$ is even and non-decreasing in $|y|$ and satisfies

$$
\left|\left\{y ; 1-\varphi^{*}(x, y)>c\right\}\right|=|\{y ; 1-|\varphi(x, y)|>c\}|
$$

for all $x$, where $|\ldots|$ denotes 1 -dimensional Lebesgue measure. (That is, $1-\varphi^{*}$ is the Steiner symmetrized function $1-|\varphi|$.) It is well known that

$$
\int_{M_{\mathscr{L}}}\left|\nabla \varphi^{*}\right|^{2} d \bar{x} \leqq \int_{M_{\mathscr{L}}}|\nabla \varphi|^{2} d \bar{x}, \int_{M_{\mathscr{L}}} V\left(\varphi^{*}\right) d \bar{x}=\int_{M_{\mathscr{L}}} V(\varphi) d \bar{x} .
$$

Hence if we extend $\varphi^{*}$ to $\tilde{M}_{\mathscr{L}}$ by using (3.1), identify points on the zero set of $\varphi^{*}$ and suitably delete double points, we obtain a minimizer of $E$, satisfying (3.1) and possessing the required symmetry properties.

Let

$$
\mathscr{H}=\mathscr{H}^{(0)}=\left\{\psi \in L_{\text {loc }}^{2}\left(\tilde{M}_{\mathscr{L}}\right) ; \psi \text { satisfies }(3.1), \psi_{\mid M_{\mathscr{L}}} \in L^{2}\left(M_{\mathscr{L}}\right)\right\}
$$

with scalar product $(\psi, \varrho)=\int_{M_{\mathscr{L}}} \psi \varrho d \bar{x}$. By (3.1) the reflexion symmetry $y \mapsto-y$ of $M_{\mathscr{L}}$ for a plane curve $\mathscr{L} \subset\{y=0\}$ induces a symmetry $\sigma$ of $\tilde{M}_{\mathscr{L}}$, splitting $\mathscr{H}=\mathscr{H}^{+} \oplus \mathscr{H}^{-}$where $\sigma_{\mid \mathscr{H}^{ \pm}}= \pm$id. For a minimizer $\varphi \in H_{\mathrm{loc}}^{1,2}\left(\tilde{M}_{\mathscr{L}}\right)$ of $E$ satisfying (3.1) let

$$
A=A(\varphi): \mathscr{D}(A) \subset \mathscr{H} \rightarrow \mathscr{H}
$$

denote the fluctuation operator associated with $\varphi$, given by the second variation of $E$

$$
\begin{aligned}
(\psi, A \varrho) & =\int_{M_{\mathscr{L}}}\left(\langle\nabla \psi, \nabla \varrho\rangle+\frac{1}{2} V^{\prime \prime}(\varphi) \psi \varrho\right) d \bar{x} \\
& =d^{2} E(\varphi)(\psi, \varrho) .
\end{aligned}
$$


Lemma 5.2. Suppose $\varphi$ is a minimizer of $E$ in $H_{\text {loc }}^{1,2}\left(\tilde{M}_{\mathscr{L}}\right)$, satisfying (3.1), and let $C_{2}=V^{\prime \prime}(1)>0$. Then the spectrum of $A=A(\varphi)$ below $C_{2}$ consists of pure point spectrum, only. In particular, if

$$
\lambda_{o}=\inf _{\psi \in \mathscr{H}} \frac{(\psi, A \psi)}{(\psi, \psi)}<C_{2},
$$

then $\lambda_{o}$ is attained at a smooth function $\psi \in \mathscr{H}$.

Moreover, if $\mathscr{L} \subset\{y=0\}, \varphi \circ \sigma=\varphi$, then the eigenspace of $A$ for any point eigenvalue $\lambda$ is spanned by eigenvectors $\psi \in \mathscr{H}^{ \pm}$.

Proof. By exponential decay of $(1-|\varphi|)$, Theorem 3.1.( $\left.3^{\circ}\right)$, the operator $A(\varphi)$ differs from the operator $A_{o}=\left(-\Delta+C_{2}\right)$ by a compact perturbation. Since the assertion of the lemma is true for $A_{o}$, it thus remains true for $A$. Finally, $\varphi \circ \sigma=\varphi$ implies that $(A \psi) \circ \sigma=A(\psi \circ \sigma)$ for any $\psi \in \mathscr{D}(A)$. Hence, if $A \psi=\lambda \psi$, also $\psi_{ \pm}=(\psi \pm \psi \circ \sigma) \in \mathscr{H}^{ \pm}$ will satisfy this relation.

Our aim is to establish the following result.

Theorem 5.3. Suppose $\mathscr{L} \subset\{y=0\}$ and let $\varphi=\varphi \circ \sigma$ be a symmetric minimizer of $E, A=A(\varphi)$ the associated fluctuation operator. Then $\varphi$ is a non-degenerate, strict minimizer of $E$ in the sense that

$$
\lambda_{o}=\inf _{\psi \in \mathscr{H}} \frac{(\psi, A \psi)}{(\psi, \psi)}>0 .
$$

Note that $\varphi_{y}=\frac{\partial}{\partial y} \varphi$ satisfies

$$
-\Delta \varphi_{y}+\frac{1}{2} V^{\prime \prime}(\varphi) \varphi_{y}=0
$$

however, $\varphi_{y} \notin H_{\text {loc }}^{1,2}\left(\tilde{M}_{\mathscr{L}}\right)$. Observe that $\varphi_{y} \geqq 0$ for $y \geqq 0, \varphi_{y} \neq 0$ on $M_{\mathscr{L}}$. Hence $\varphi_{y}>0$ for $y>0$, on $M_{\mathscr{L}}$, by the maximum principle. A similar property holds for eigenfunctions of $A(\varphi)$ with eigenvalue $\lambda_{o}$. Let

$$
\mathscr{H}_{o}=\left\{\psi \in \mathscr{H} ; A \psi=\lambda_{o} \psi\right\}=\mathscr{H}_{o}^{+} \oplus \mathscr{H}_{o}^{-},
$$

where $\mathscr{H}_{o}^{ \pm}=\mathscr{H}_{o} \cap \mathscr{H}^{ \pm}$.

Lemma 5.4. Let $\psi_{ \pm} \in \mathscr{H}_{o}^{ \pm} \backslash\{0\}$ be eigenfunctions for $\lambda_{o}$. Then $\psi_{ \pm} \neq 0$ for $y>0$ on $M_{\mathscr{L}}$.

Proof. Replace $\psi_{ \pm}$by $\hat{\psi}_{ \pm}$given by $\left|\psi_{ \pm}\right|$on $\mathbb{R}^{3} \cap\{y>0\}$ and by $\pm\left|\psi_{ \pm}\right|$on $\mathbb{R}^{3}$ $\cap\{y<0\}$. Extend $\hat{\psi}_{ \pm}$to $\tilde{M}_{\mathscr{L}}$ by using (3.1). Since $\psi_{ \pm}$satisfied (3.1), $\hat{\psi}_{ \pm} \in H_{\text {loc }}^{1,2}\left(\tilde{M}_{\mathscr{L}}\right)$ and there holds

$$
\left(\hat{\psi}_{ \pm}, A \hat{\psi}_{ \pm}\right)=\left(\psi_{ \pm}, A \psi_{ \pm}\right),\left(\hat{\psi}_{ \pm}, \hat{\psi}_{ \pm}\right)=\left(\psi_{ \pm}, \psi_{ \pm}\right)
$$

Hence $\hat{\psi}_{ \pm} \in \mathscr{H}_{o}$; in particular, $\hat{\psi}_{ \pm}$satisfies

$$
-\Delta \hat{\psi}_{ \pm}+\frac{1}{2} V^{\prime \prime}(\varphi) \hat{\psi}_{ \pm}=\lambda_{o} \hat{\psi}_{ \pm},
$$

and thus $\hat{\psi}_{ \pm} \in C^{\infty}\left(\tilde{M}_{\mathscr{L}}\right)$. Finally, by the maximum principle, applied to (5.2), $\hat{\psi}_{ \pm}>0$ for $y>0$ on $M_{\mathscr{L}}$, which implies the claim. 
Remark. Multiplying by a suitable constant, if necessary, we may assume that

$$
\varphi \psi_{+} \geqq 0, \varphi_{y} \psi_{-} \geqq 0 \quad \text { on } \quad \tilde{M}_{\mathscr{L}},
$$

for $\psi_{ \pm} \in \mathscr{H}_{0}^{ \pm} \backslash\{0\}$, with equality at most for $y=0$.

Proof of Theorem 5.3. Suppose by contradiction that $\lambda_{o}=0$. Let $\psi_{ \pm} \in \mathscr{H}_{o}^{ \pm}$, satisfying

$$
-\Delta \psi_{ \pm}+\frac{1}{2} V^{\prime \prime}(\varphi) \psi_{ \pm}=0 .
$$

(i) To see that $\mathscr{H}_{0}^{+}=\{0\}$ we argue by contradiction. Suppose $\psi_{+}>0$ in $\{y>0\}$. Multiply (3.2) - for $\varepsilon=1$ - by $\psi_{+}$, (5.4) by $\varphi$ and subtract. Upon integrating by parts we obtain

$$
\begin{aligned}
0 & =\int_{M_{\mathscr{L}}}\left[\left(-\Delta \varphi \psi_{+}+\Delta \psi_{+} \varphi\right)+\frac{1}{2} V^{\prime}(\varphi) \psi_{+}-\frac{1}{2} V^{\prime \prime}(\varphi) \varphi \psi_{+}\right] d \bar{x} \\
& =\frac{1}{2} \int_{M_{\mathscr{L}}}\left[\left(V^{\prime}(\varphi)-V^{\prime \prime}(\varphi) \varphi\right) \psi_{+}\right] d \bar{x} \\
& =\int_{M_{\mathscr{L}} \cap\{y>0\}}\left[\left(V^{\prime}(\varphi)-V^{\prime \prime}(\varphi) \varphi\right) \psi_{+}\right] d \bar{x}<0
\end{aligned}
$$

by reflexion symmetry and assumption (V4), Sect. 3. This yields the desired contradiction.

(ii) We show that also $\mathscr{H}_{o}^{-}=0$. Again suppose $\psi_{-}>0$ in $\{y>0\}$. Multiply (5.1) by $\psi_{-},(5.4)$ by $\varphi_{y}$ and subtract. Integrating by parts over

$$
M_{\mathscr{L}}^{\varepsilon}=M_{\mathscr{L}} \cap\{\bar{x} ; \operatorname{dist}(\bar{x}, \mathscr{L}) \geqq \varepsilon\}
$$

we obtain the relation

$$
\begin{aligned}
0 & =\int_{\mathcal{M}_{\mathcal{E}}^{\varepsilon}}\left(-\Delta \varphi_{y} \psi_{-}+\Delta \psi_{-} \varphi_{y}\right) d \bar{x} \\
& =\int_{\partial \mathcal{M}_{\tilde{I}}^{\varepsilon}}\left(\partial_{\nu} \psi_{-} \varphi_{y}-\partial_{\nu} \varphi_{y} \psi_{-}\right) d o,
\end{aligned}
$$

where $v$ denotes the unit normal on $\partial M_{\mathscr{L}}^{\varepsilon}$ pointing towards $\mathscr{L}$.

As in the proof of Theorem 3.2 we locally represent $\psi_{-}$and $\varphi$ in parallel coordinates $(z, t)$ based on $\mathscr{L}$, where $z=z_{1}+i z_{2} \in \mathbb{C} \cong \mathbb{R}^{2}$, by mappings

$$
\tilde{\varphi}(z, t)=\varphi\left(S\left(z^{2}, t\right)\right), \quad \tilde{\psi}_{-}=\psi_{-}\left(S\left(z^{2}, t\right)\right),
$$

solving equations of type (3.7).

By our regularity result Lemma 3.3, applied to these maps, for fixed $t$ we have expansions

$$
\begin{aligned}
\tilde{\varphi}(z, t) & =a_{11} z_{1}+a_{12} z_{2}+a_{21} z_{1}^{2}+a_{22} z_{1} z_{2}+a_{23} z_{2}^{2}+\ldots, \\
\tilde{\psi}_{-}(z, t) & =b_{11} z_{1}+b_{12} z_{2}+b_{21} z_{1}^{2}+b_{22} z_{1} z_{2}+b_{23} z_{2}^{2}+\ldots
\end{aligned}
$$

Hence, introducing polar coordinates $(\varrho, \theta)$ in a disc around $S(0, t)$ in a plane orthogonal to $\mathscr{L}$, we also have expansions

$$
\begin{aligned}
\varphi_{y}(\varrho, \theta, t) & =\left(\alpha \cos \left(\frac{\theta}{2}\right)+\beta \sin \left(\frac{\theta}{2}\right)\right) \varrho^{-1 / 2}+O\left(\varrho^{1 / 2}\right), \\
\psi_{-}(\varrho, \theta, t) & =\left(\gamma \cos \left(\frac{\theta}{2}\right)+\delta \sin \left(\frac{\theta}{2}\right)\right) \varrho^{1 / 2}+0\left(\varrho^{3 / 2}\right) .
\end{aligned}
$$


Condition (5.3) now requires that for some constant $\mu=\mu(t)>0$ we have $\alpha=\mu \gamma$, $\beta=\mu \delta$; that is

$$
\psi_{-}=\mu \varrho \varphi_{y}+O\left(\varrho^{3 / 2}\right) .
$$

Inserting this relation into (5.5) yields

$$
0=\int_{\partial M_{\mathscr{E}}^{\varepsilon}} \mu\left|\varphi_{y}\right|^{2} d o+0(\varepsilon),
$$

which is positive for small $\varepsilon>0$, giving the desired contradiction.

For general curves $\mathscr{L}$, our results are only fragmentary. Very likely, asymptotic stability properties of minimizers of $E_{\varepsilon}$ for small $\varepsilon$ can be inferred from a detailed study of the second variation of $E_{\varepsilon}\left(\varphi_{\varepsilon}\right)$ with respect to variations of the independent variables, and relating this to the second variation of area of the limiting minimal surface. A crude estimate to that effect can be obtained as follows:

Let $\varrho \in C_{o}^{\infty}\left(\tilde{M}_{\mathscr{L}}\right)$ satisfy (3.1) and compute the second variation of $E=E_{1}$ at $\varphi=\varphi_{1}$ in direction $\psi=|\nabla \varphi| \varrho$. Note that $\psi \in H_{\text {loc }}^{1,2}\left(\tilde{M}_{\mathscr{L}}\right)$, and $\psi$ also satisfies (3.1). Moreover, a large class of such mappings $\psi$ can be represented in this form. Now,

$$
\begin{aligned}
\Lambda:=d^{2} E(\varphi)(|\nabla \varphi| \varrho,|\nabla \varphi| \varrho)= & \int_{M_{\mathscr{L}}}\left(|\nabla(|\nabla \varphi| \varrho)|^{2}+\frac{1}{2} V^{\prime \prime}(\varphi)|\nabla \varphi|^{2} \varrho^{2}\right) d x \\
= & \int_{M_{\mathscr{L}}}\left[|\nabla(|\nabla \varphi|)|^{2} \varrho^{2}+\frac{1}{2} \nabla\left(|\nabla \varphi|^{2}\right) \nabla\left(\varrho^{2}\right)+|\nabla \varphi|^{2}|\nabla \varrho|^{2}\right. \\
& \left.+\frac{1}{2} V^{\prime \prime}(\varphi)|\nabla \varphi|^{2} \varrho^{2}\right] d x .
\end{aligned}
$$

On the other hand, differentiating (3.2) and multiplying by $\nabla \varphi \varrho^{2}$, we obtain

$$
-\Delta\left(\frac{|\nabla \varphi|^{2}}{2}\right) \varrho^{2}+\left|\nabla^{2} \varphi\right|^{2} \varrho^{2}+\frac{V^{\prime \prime}(\varphi)}{2}|\nabla \varphi|^{2} \varrho^{2}=0 \text {. }
$$

Integrating and subtracting this expression from the previous one we have

$$
\Lambda=\int_{M_{\mathscr{L}}}\left[|\nabla \varphi|^{2}|\nabla \varrho|^{2}-\left(\left|\nabla^{2} \varphi\right|^{2}-|\nabla(|\nabla \varphi|)|^{2}\right) \varrho^{2}\right] d x .
$$

Now let $v=\frac{\nabla \varphi}{|\nabla \varphi|}$. Then

$$
|\nabla v|^{2}=\left|\frac{\nabla^{2} \varphi}{|\nabla \varphi|}-\frac{\nabla|\nabla \varphi| \nabla \varphi}{|\nabla \varphi|^{2}}\right|^{2}=\frac{\left|\nabla^{2} \varphi\right|^{2}-|\nabla(|\nabla \varphi|)|^{2}}{|\nabla \varphi|^{2}}
$$

whence

$$
\Lambda=\int_{M_{\mathscr{L}}}|\nabla \varphi|^{2}\left(|\nabla \varrho|^{2}-|\nabla v|^{2} \varrho^{2}\right) d x
$$

equals the weighted mean of the second variations of area of the level surfaces $\varphi=$ const with weight $|\nabla \varphi|^{2}$, see Giusti [29; (10.30), p. 120].

However, we regard this only as a first step towards the solution of the question of stability of minimizers of $E_{\varepsilon}$ in general. In order to deduce stability of $\varphi_{\varepsilon}$ from stability of a limiting minimal surface, represented by a map $\varphi_{o}$, via (5.4), it seems that deeper insights into the speed and nature of convergence $\varphi_{\varepsilon} \rightarrow \varphi_{o}$ are needed.

To conclude, we wish to remark that all methods developed in Sects. 2 through 5 can also be applied to solve problem I described in Sect. 1, where $\mathbb{R}^{3}$ is replaced 
by $\mathbb{R}^{2}$ and $\mathscr{L}$ by a set of points $\left\{x_{1}, \ldots, x_{2 n}\right\}$. In fact, problem $\mathrm{I}$ is somewhat simpler than the three-dimensional problem studied here.

Acknowledgements. This paper is an attempt to summarize discussions at various occasions throughout the last two years on questions in field theory and statistical mechanics and mathematically rigorous ways of approaching them. In the beginning, we received some highly valuable input also from U. Abresch and H.-W. Alt, that we gratefully acknowledge. Moreover, one of us (J.F.) thanks P.-A. Marchetti for numerous discussions on the topics discussed in Sect. 1.

\section{References}

1. Mermin, N.D.: Rev. Mod. Phys. 51, 591 (1979) Michel, L.: Rev. Mod. Phys. 52, 617 (1980)

2. Kramers, H.A., Wannier, G.H.: Phys. Rev. 60, 252-262 (1941)

Wegner, F.: J. Math. Phys. 12, 2259 (1971)

3. Wilson, K.G.: Phys. Rev. D 10, 2445 (1974)

4. Glimm, J., Jaffe, A.: Quantum physics. A functional integral point of view. Berlin, Heidelberg, New York: Springer 1987

5. Glimm, J., Jaffe, A., Spencer, T.: Ann. Phys. (NY) 101, 610 (1976); 101, 631 (1976)

6. Fröhlich, J.: Commun. Math. Phys. 47, 269 (1976)

Bellissard, J., Fröhlich, J., Gidas, B.: Commun. Math. Phys. 60, 37 (1978)

7. Hepp, K.: Commun. Math. Phys. 35, 265 (1974)

Eckmann, J.-P.: Lett. Math. Phys. 1, 387 (1977)

8. Nelson, E.: J. Funct. Anal. 12, 97 (1973)

9. Osterwalder, K., Schrader, R.: Commun. Math. Phys. 31, 83 (1973); 42, 281 (1975)

Glaser, V.: Commun. Math. Phys. 37, 257 (1974)

See also: Constructive quantum field theory. Velo, G., Wightman, A.S. (eds.). Berlin, Heidelberg, New York: Springer 1973

10. Fröhlich, J.: In: Recent Developments in Gauge Theory, (Cargèse 1979). 't Hooft, G. et al. (eds.). New York: Plenum Press 1980

Marino, E.C., Swieca, J.A.: Nucl. Phys. B 170 [FS 1], 175 (1980)

Marino, E.C., Schroer, B., Swieca, J.A.: Nucl. Phys. B 200 [FS 4], 473 (1982)

11. Fröhlich, J., Marchetti, P.A.: Commun. Math. Phys. 116, 127 (1988)

12. Jaffe, A., Taubes, C.H.: Vortices and Monopoles: Structure of Static Gauge Theories. Basel, Boston: Birkhäuser 1980

13. Fröhlich, J., Marchetti, P.A.: Commun. Math. Phys. 121, 177 (1989)

14. Balaban, T.: Commun. Math. Phys. 85, 603 (1982); 86, 555 (1982); 88, 411 (1983); 89, 571 (1983) Federbush, P.: Commun. Math. Phys. 107, 319 (1987)

King, C.: Commun. Math. Phys. 102, 649 (1986); 103, 323 (1986)

15. de Gennes, P.G.: Superconductivity of metals and alloys. New York: Benjamin 1966

16. Brézis, H., Coron, J.M., Lieb, E.: Harmonic maps with defects. Commun. Math. Phys. 107, 649-705 (1986)

17. Almgren, F., Browder, F., Lieb, E.: Co-area, liquid crystals, and minimal surfaces. In: DD7-a selection of papers. Berlin, Heidelberg, New York: Springer 1987

18. Adams, R.A.: Sobolev spaces. New York: Academic Press 1975

19. Giaquinta, M.: Multiple integrals in the calculus of variations and non linear elliptic systems. Princeton, NJ: Princeton University Press 1983

20. Morrey, C.B.: Multiple integrals in the calculus of variations. Berlin, Heidelberg, New York: Springer 1966

21. Gilbarg, D., Trudinger, N.S.: Elliptic partial differential equations of second order. Berlin, Heidelberg, New York: Springer 1983

22. Modica, L.: The gradient theory of phase transitions and the minimal interface criterion. Arch. Rat. Mech. Anal. 98, 123-142 (1986) 
23. Modica, L., Mortola, S.: Un esempio di $\Gamma^{-}$-convergenza. Boll. U.M.I. (5) 14-B, 285-299 (1977)

24. Gurtin, M.E.: Some results and conjectures in the gradient theory of phase transitions. Inst. for Math. and its Appl., University of Minnesota, Preprint No. 32, 156 (1985)

25. Kohn, R.V., Sternberg, P.: Local minimizers and singular perturbations. Proc. R. Soc. Edinb. $111 \mathrm{~A}, 69-84$ (1989)

26. Anzellotti, G., Baldo, S., Visintin, A.: Asymptotic behavior of the Landau-Lipschitz model of ferromagnetism. Preprint (Povo; 1989)

27. Baldo, S.: Minimal interface criterion for phase transitions in mixtures of Cahn-Milliard fluids. Preprint (Pisa; 1988)

28. Luckhaus, S., Modica, L.: The Gibbs Thompson relation within the gradient theory of phase transitions. Arch. Rat. Mech. Anal. 107, 71-83 (1989)

29. Giusti, E.: Minimal surfaces and functions of bounded variation. Basel, Boston: Birkhäuser 1984

30. Modica, L.: Geometry of level sets of entire solutions of semilinear elliptic equations. Oberwolfach Tagungsbericht 27 (1988)

31. Bronsard, L., Kohn, R.V.: Motion by mean curvature as the singular limit of GinzburgLandau dynamics. Preprint (Brown University 1989)

32. Carr, J., Pego, R.: Very slow phase separation in one dimension. In: Conf. on Phase Transitions (ed.) Rasele, M., Nice (1987)

33. Alikakos, N., Bates, P.W., Fusco, G.: Slow motion for Cahn-Hilliard equation in one space dimension. Preprint (Rome 1989)

34. Hardt, R., Simon, L.: Boundary regularity and embedded solutions for the oriented Plateau problem. Ann. Math. 110, 439-486 (1979)

Communicated by A. Jaffe

Received March 16, 1990 